\title{
Poisson brackets and structure of nongraded Hamiltonian Lie alge- bras related to locally-finite derivations
}

(to Appear in Canadian J. Math.)

\section{Yucai Su}

Department of Mathematics, Shanghai Jiaotong University, Shanghai 200030, P. R. China

(e-mail: ycsu@sjtu.edu.cn)

\begin{abstract}
Xu}$ introduced a class of nongraded Hamiltonian Lie algebras. These Lie algebras have a Poisson bracket structure. In this paper, the isomorphism classes of these Lie algebras are determined by employing a "sandwich" method and by studying some features of these Lie algebras. It is obtained that two Hamiltonian Lie algebras are isomorphic if and only if their corresponding Poisson algebras are isomorphic. Furthermore, the derivation algebras and the second cohomology groups are determined.
\end{abstract}

Mathematical Subject Classification (1991): 17B40, $17 B 65$

\section{Introduction}

A Lie algebra $(\mathcal{A},[\cdot, \cdot])$ is called to have a Poisson bracket structure if there exists a commutative associative algebra structure $(\mathcal{A}, \cdot)$ such that the compatibility condition holds:

$$
[u, v \cdot w]=[u, v] \cdot w+v \cdot[u, w] \text { for } u, v, w \in \mathcal{A}
$$

The algebra $(\mathcal{A}, \cdot,[\cdot, \cdot])$ with two algebraic structures is also called a Poisson algebra. Poisson bracket structures have many applications in areas of mathematics and physics; they are fundamental algebraic structures on phase spaces in classical mechanics; they are also the main objects in symplectic geometry (cf. [Z]).

Let $\mathbb{F}$ be a field of characteristic zero. A Lie algebra $\mathcal{A}$ is called graded if $\mathcal{A}=\oplus_{\alpha \in \Gamma} \mathcal{A}_{\alpha}$ is a $\Gamma$-graded $\mathbb{F}$-vector space for some abelian group $\Gamma$ such that

$$
\operatorname{dim} \mathcal{A}_{\alpha}<\infty, \quad\left[\mathcal{A}_{\alpha}, \mathcal{A}_{\beta}\right] \subset \mathcal{A}_{\alpha+\beta} \quad \text { for } \quad \alpha, \beta \in \Gamma .
$$

A classical Poisson algebra $\mathcal{P}(\ell)$ is a polynomial algebra $\mathcal{A}=\mathbb{F}\left[t_{1}, t_{2}, \cdots, t_{2 \ell}\right]$ in $2 \ell$ variables with the Lie bracket

$$
[f, g]=\sum_{i=1}^{\ell}\left(\partial_{t_{i}}(f) \partial_{t_{\ell+i}}(g)-\partial_{t_{\ell+i}}(f) \partial_{t_{i}}(g)\right) \text { for } \quad f, g \in \mathcal{A}
$$

where $\partial_{t_{i}}$ stands for partial derivative $\frac{\partial}{\partial t_{i}}$. Define

$$
\mathcal{P}(\ell)_{n}=\left\{t_{1}^{n_{1}} t_{2}^{n_{2}} \cdots t_{2 \ell}^{n_{2 \ell}} \mid n_{i} \in \mathbb{N}, \sum_{i=1}^{2 \ell} n_{i}=n+2\right\} \quad \text { for } \quad-2 \leq n \in \mathbb{Z},
$$

then $\mathcal{P}(\ell)$ is a $\mathbb{Z}$-graded algebra $\mathcal{P}(\ell)=\oplus_{n \in \mathbb{Z}} \mathcal{P}(\ell)_{n}$. When we consider only its Lie algebra structure, this Lie algebra is denoted by $\mathcal{H}(\ell)$. Then $\mathcal{H}(\ell)$ (or the simple Lie algebra $[\mathcal{H}(\ell), \mathcal{H}(\ell)] / \mathbb{F}$ ) is a classical Lie algebra of Cartan type $H$ (also called a Hamiltonian Lie 
algebra) [K1, K2]. Generalizations of graded Hamiltonian Lie algebras have been studied in $[\mathrm{O}, \mathrm{OZ}]$.

Nongraded Lie algebras appear naturally in the theory of vertex algebras and their multivariable analogues, they play important roles in mathematical physics. Xu [X2] constructed a family of in general nongraded Hamiltonian Lie algebras based on certain derivation-simple algebras and locally finite derivations (we refer to [SXZ] for the classification of derivationsimple algebras). In $[\mathrm{SX}], \mathrm{Xu}$ and the author of this paper determined the isomorphism classes of Poisson algebras constructed in [X2] (two Poisson algebras are called isomorphic if there exists an isomorphism which preserves both associative algebra structure and Lie algebra structure). However, the structure theory of the Hamiltonian Lie algebras in general does not seem to be well-developed. Since the Poisson algebras have two compatible algebraic structures while the Hamiltonian Lie algebras only have a Lie algebraic structure, the problem of determination of the isomorphism classes of Hamiltonian Lie algebras is thus more complicated, and one can see that some special treatments are needed in order to determine their isomorphism classes.

In [OZ], Osborn and Zhao determined the isomorphism classes of the graded Hamiltonian Lie algebras under certain finiteness condition on the skew-symmetric $\mathbb{Z}$-bilinear forms $\phi_{0}$. They used the "derivation method" to determine the isomorphism classes of the Hamiltonian Lie algebras, mainly, they first determined the derivation algebras of the Lie algebras in order to obtain their isomorphism theorem. In this paper, we shall determine the isomorphism classes of in general nongraded Hamiltonian Lie algebras $\mathcal{H}(\underline{\ell}, \Gamma)$, where $\underline{\ell}$ is a 7 -tuple of nonnegative integers and $\Gamma$ is some free abelian group, which correspond to the Lie algebras in $[\mathrm{SX}]$ with the skew-symmetric $\mathbb{Z}$-bilinear form $\phi$ being zero and $\ell_{4}=0$. The reason we choose $\phi=\ell_{4}=0$ is that the Hamiltonian Lie algebras look more natural and more explicit, and are therefore easier for application, and also they are general enough to cover already most interesting cases (see $\S 2)$. The Hamiltonian Lie algebras considered in [OZ] in case $\phi_{0}=0$ are the cases of the Hamiltonian Lie algebras $[\mathcal{H}(\underline{\ell}, \Gamma), \mathcal{H}(\underline{\ell}, \Gamma)] / \mathbb{F}$ with $\underline{\ell}=(\ell, 0, \cdots, 0)$.

Unlike the graded case, where the sets of $a d$-locally finite elements and ad-locally nilpotent elements can be determined, in the nongraded case, the determination of the sets of ad-locally finite elements and ad-locally nilpotent elements seems to be un-achievable. Here, we use a "sandwich" method to estimate them (see Lemma 3.1). By studying some important features of the Hamiltonian Lie algebras (Lemma 3.4), we are able to obtain the isomorphism theorem without the need to know the structure of their derivation algebras. We obtain

Main Theorem. Two Hamiltonian Lie algebras are isomorphic if and only if their corresponding Poisson algebras are isomorphic.

In Section 2, we shall rewrite the presentations of the above-mentioned Hamiltonian Lie algebras up to certain obvious isomorphisms, which we call normalized forms. Then we shall prove the main theorem in Section 3. In Section 4, we shall use a different method from those in $[\mathrm{F}, \mathrm{OZ}]$ to determine the derivation algebras of the Hamiltonian Lie algebras. The reason we determine the derivation algebras after the determination of the isomorphism classes is that 
we want to emphasize that the determination of the isomorphism classes does not depend on the determination of the derivation algebras. Then in the final section, we shall determine the second cohomology groups of the Hamiltonian Lie algebras (the second cohomology groups of the Hamiltonian Lie algebras considered in [OZ] was determined by Jia [J]).

Acknowledgements. The author would like to thank Dr. Xiaoping Xu for suggesting the investigation of this problem and for instructions, Professor Kaiming Zhao for helpful discussions. Part of this research was carried out during the author's visit to Academy of Mathematics and Systems Sciences, Chinese Academy of Sciences, he wishes to thank the Academy for hospitality and support. This research was Supported by a NSF grant no. 10171064 of China and two research grants from Ministry of Education of China.

\section{Normalized Forms}

Before we present the normalized forms of the Hamiltonian Lie algebras, to better understand general Hamiltonian Lie algebras, we first explain how one can generalize the classical Hamiltonian Lie algebras $\mathcal{H}(\ell)$ defined in (1.3).

For convenience, we denote

$$
\bar{i}=i+\ell \text { for } \quad 1 \leq i \leq \ell
$$

The constructional ingredients of the classical Hamiltonian Lie algebra $\mathcal{H}(\ell)$ are the pairs $(\mathcal{A}, \mathcal{D})$ consisting of the polynomial algebra

$$
\mathcal{A}=\mathbb{F}\left[t_{1}, t_{\overline{1}}, \cdots, t_{\ell}, t_{\bar{\ell}}\right]
$$

and a finite dimensional space $\mathcal{D}=\operatorname{span}\left\{\partial_{t_{i}}, \partial_{t_{\bar{i}}} \mid 1 \leq i \leq \ell\right\}$ of commuting locally finite derivations. The derivations $\partial_{t_{i}}=\frac{\partial}{\partial t_{i}}$ are called down-grading operators by its obvious meaning for $1 \leq i \leq 2 \ell$. Then the type of derivation pairs $\left\{\left(\partial_{t_{i}}, \partial_{t_{\bar{i}}}\right) \mid 1 \leq i \leq \ell\right\}$ for $\mathcal{H}(\ell)$ is

$$
(d, d)
$$

where "d" stands for down-grading operators.

If we replace the polynomial algebra by the Laurant polynomial algebra

$$
\mathcal{A}=\mathbb{F}\left[x_{1}^{ \pm 1}, x_{\overline{1}}^{ \pm 1}, \cdots, x_{\ell}^{ \pm 1}, x_{\bar{\ell}}^{ \pm 1}\right]
$$

and rewrite (1.3) as

$$
[f, g]=\sum_{p=1}^{\ell}\left(x_{p} x_{\bar{p}}\right)^{-1}\left(\partial_{p}^{*}(f) \partial_{\bar{p}}^{*}(g)-\partial_{\bar{p}}^{*}(f) \partial_{p}^{*}(g)\right) \quad \text { for } \quad f, g \in \mathcal{A},
$$

where $\partial_{p}^{*}$ stands for $x_{p} \frac{\partial}{\partial x_{p}}$ for $1 \leq p \leq 2 \ell$, then we obtain a Hamiltonian Lie algebra, denoted by $\overline{\mathcal{H}}(\ell)$. Now the derivations $\partial_{p}^{*}$ are called grading operators by its obvious meaning, and the type of derivation pairs $\left\{\left(\partial_{p}^{*}, \partial_{\bar{p}}^{*}\right) \mid 1 \leq p \leq \ell\right\}$ for $\overline{\mathcal{H}}(\ell)$ is then

$$
(g, g)
$$


where "g" stands for grading operators.

Furthermore, we can replace $\mathcal{A}$ by a semigroup algebra which is the tensor product of a Laurant polynomial algebra (2.4) and a polynomial algebra (2.2):

$$
\mathcal{A}=\mathbb{F}\left[x_{1}^{ \pm 1}, t_{1}, x_{\overline{1}}^{ \pm 1}, t_{\overline{1}}, \cdots, x_{\ell}^{ \pm 1}, t_{\ell}, x_{\bar{\ell}}^{ \pm 1}, t_{\bar{\ell}}\right]
$$

and replace $\partial_{p}^{*}$ by $\partial_{p}=\partial_{p}^{*}+\partial_{t_{p}}$ for $1 \leq p \leq 2 \ell$, then (2.5) defines a Hamiltonian Lie algebra, denoted by $\mathcal{H}(\ell)$. The derivation $\partial_{p}$ are called mixed operator, and the type of derivation pairs $\left\{\left(\partial_{p}, \partial_{\bar{p}}\right) \mid 1 \leq p \leq \ell\right\}$ for $\widehat{\mathcal{H}}(\ell)$ is now

$$
(m, m)
$$

where "m" stands for mixed operators.

In the examples above, we can generally denote a monomial as

$$
x^{\alpha, \underline{i}}=x_{1}^{\alpha_{1}} x_{\overline{1}}^{\alpha_{\overline{1}}} \cdots x_{\ell}^{\alpha_{\ell}} x_{\bar{\ell}}^{\alpha_{\bar{\ell}}} t_{1}^{i_{1}} t_{\overline{1}}^{i_{\overline{1}}} \cdots t_{\ell}^{i_{\ell}} t_{\bar{\ell}}^{i_{\bar{\ell}}}
$$

for

$$
\alpha=\left(\alpha_{1}, \alpha_{\overline{1}}, \cdots, \alpha_{\ell}, \alpha_{\bar{\ell}}\right) \in \Gamma, \quad \underline{i}=\left(i_{1}, i_{\overline{1}}, \cdots, i_{\ell}, i_{\bar{\ell}}\right) \in \mathcal{J},
$$

where $\Gamma$ is an additive subgroup of $\mathbb{F}^{2 \ell}$ such that $\Gamma=\{0\}$ in the case of $\mathcal{H}(\ell)$ (where there are no nonzero grading operators), and $\Gamma=\mathbb{Z}^{2 \ell}$ in the cases of $\overline{\mathcal{H}}(\ell)$ and $\widehat{\mathcal{H}}(\ell)$ (where there are nonzero grading operators), and where $\mathcal{J}$ is some semi-subgroup of $\mathbb{N}^{2 \ell}$ such that $\mathcal{J}=\mathbb{N}^{2 \ell}$ in the cases of $\mathcal{H}(\ell)$ and $\widehat{\mathcal{H}}(\ell)$ (where there are nonzero down-grading operators), and $\mathcal{J}=\{0\}$ in the case of $\overline{\mathcal{H}}(\ell)$ (where there are no nonzero down-grading operators). In all three cases, we can define operators $\partial_{p}^{*}=x_{p} \frac{\partial}{\partial x_{p}}, \partial_{t_{p}}=\frac{\partial}{\partial t_{p}}$ and $\partial_{p}=\partial_{p}^{*}+\partial_{t_{p}}$ such that $\partial_{p}^{*}=0$ in the case of $\mathcal{H}(\ell)$ and $\partial_{t_{p}}=0$ in the case of $\overline{\mathcal{H}}(\ell)$.

With the above examples in mind, we can now give generalizations of the Hamiltonian Lie algebras as follows.

First for convenience, for $m, n \in \mathbb{Z}$, we denote

$$
\overline{m, n}= \begin{cases}\{m, m+1, \cdots, n\} & \text { if } m \leq n \\ \emptyset & \text { otherwise }\end{cases}
$$

We shall construct a semigroup algebra $\mathbb{F}[\Gamma \times \mathcal{J}](\mathrm{cf} .(2.7))$, where $\Gamma$ is some free abelian subgroup of an $\mathbb{F}$-vector space $\mathbb{F}^{n}$ and $\mathcal{J}$ is some semi-subgroup of $\mathbb{N}^{n}$, and construct 7 groups of derivation pairs $\left\{\left(\partial_{p}, \partial_{\bar{p}}\right) \mid p \in I_{i}\right\}$ for $i \in \overline{1,7}$, where $I_{i}$ are some indexing sets such that if we denote each type of derivation pairs $\left\{\left(\partial_{p}, \partial_{\bar{p}}\right) \mid p \in I_{i}\right\}$ by $\left(T_{i}, T_{\bar{i}}\right)$ for $i \in \overline{1,7}$, then the types of derivation pairs in the order of the groups $\left\{\left(\partial_{p}, \partial_{\bar{p}}\right) \mid p \in I_{i}\right\}$ for $i \in \overline{1,7}$ are

$$
\begin{array}{llll}
\left(T_{1}, T_{\overline{1}}\right)=(g, g), & \left(T_{2}, T_{\overline{2}}\right)=(m, g), & \left(T_{3}, T_{\overline{3}}\right)=(m, g), & \left(T_{4}, T_{\overline{4}}\right)=(m, m), \\
\left(T_{5}, T_{\overline{5}}\right)=(g, d), & \left(T_{6}, T_{\overline{7}}\right)=(m, d), & \left(T_{7}, T_{\overline{7}}\right)=(d, d) . &
\end{array}
$$

Then we shall see that (2.3), (2.6) and (2.8) correspond respectively to the three special cases: (i) $I_{7}=\overline{1, \ell}$ and $I_{i}=\emptyset$ if $i \neq 7$, (ii) $I_{1}=\overline{1, \ell}$ and $I_{i}=\emptyset$ if $i \neq 1$, and (iii) $I_{4}=\overline{1, \ell}$ and $I_{i}=\emptyset$ if $i \neq 4$. 
To construct, we let

$$
\underline{\ell}=\left(\ell_{1}, \cdots, \ell_{7}\right) \in \mathbb{N}^{7} \backslash\{0\} .
$$

Set

$$
\begin{aligned}
& \iota_{0}=0, \quad \iota_{i}=\ell_{1}+\ell_{2}+\ldots+\ell_{i}, \quad i \in \overline{1,7}, \\
& I_{i, j}=\overline{\iota_{i-1}+1, \iota_{j}} \text { for } i, j \in \overline{1,7}, \quad i \leq j .
\end{aligned}
$$

Denote

$$
I_{i}=I_{i, i}, \quad I=I_{1,7}, \quad J=\overline{1,2 \iota_{7}} .
$$

Define the map ${ }^{-}: J \rightarrow J$ by

$$
\bar{p}=\left\{\begin{array}{lll}
p+\iota_{7} & \text { if } & p \in \overline{1, \iota_{7}}, \\
p-\iota_{7} & \text { if } & p \in \overline{\iota_{7}+1,2 \iota_{7}},
\end{array}\right.
$$

(cf. (2.1)). For any subset $K$ of $\overline{1,2 \iota_{7}}$, we denote

$$
\bar{K}=\{\bar{p} \mid p \in K\} .
$$

In particular, we have $J=I \cup \bar{I}$. Set

$$
J_{i}=I_{i} \cup \bar{I}_{i}, \quad J_{i, j}=I_{i, j} \cup \bar{I}_{i, j} \quad \text { for } \quad i, j \in \overline{1,7}, \quad i \leq j .
$$

Let $\mathbb{F}$ be a field of characteristic zero. We write an element $\alpha$ of $\mathbb{F}^{2 \iota_{7}}$ in the form

$$
\alpha=\left(\alpha_{1}, \alpha_{\overline{1}}, \cdots, \alpha_{\iota 7}, \alpha_{\overline{\iota 7}}\right) \quad \text { with } \quad \alpha_{p} \in \mathbb{F},
$$

(cf. $(2.10))$. Set

$$
\varepsilon_{p}=\left(\delta_{1, p}, \delta_{\overline{1}, p}, \cdots, \delta_{\iota_{7}, p}, \delta_{\overline{l_{7}, p}}\right) \in \mathbb{F}^{2 \iota_{7}} \text { for } p \in J .
$$

For $\alpha \in \mathbb{F}^{2 \iota_{7}}$ and $K \subset J$, we use $\alpha_{K}$ to denote the vector in $\mathbb{F}^{|K|}$ (where $|K|$ is the size of $K$ ), obtained from $\alpha$ by deleting all the coordinate $\alpha_{p}$ with $p \in J \backslash K$; for instance,

$$
\alpha_{\{1,3\}}=\left(\alpha_{1}, \alpha_{3}\right) \in \mathbb{F}^{2}, \quad \alpha_{\{1, \overline{2}, \overline{3}\}}=\left(\alpha_{1}, \alpha_{\overline{2}}, \alpha_{\overline{3}}\right) \in \mathbb{F}^{3} .
$$

Sometimes, when the context is clear, we also use $\alpha_{K}$ to denote the vector in $\mathbb{F}^{2 \iota 7}$ by putting its $p$ th coordinate to be zero for $p \in J \backslash K$.

We fix a set $\left\{\sigma_{p} \mid p \in J\right\}$ of elements in $\mathbb{F}^{2 \iota_{7}}$ as follows:

$$
\sigma_{p}=\left\{\begin{array}{lll}
\varepsilon_{p}+\varepsilon_{\bar{p}} & \text { if } & p \in I_{1} \cup I_{3,4}, \\
\varepsilon_{p} & \text { if } & p \in I_{2}, \\
0 & \text { if } & p \in I_{5,7},
\end{array}\right.
$$

and $\sigma_{\bar{p}}=\sigma_{p}$. Using the notations (2.9) and (2.23), the factor $\left(x_{p} x_{\bar{p}}\right)^{-1}$ appears in (2.5) is simply $x^{-\sigma_{p}}$ if $p \in I_{1}$. If we re-denote $x_{p}^{-1}$ by $x_{p}$ (and $x_{p}$ by $\left.x_{p}^{-1}\right)$, then the factor $\left(x_{p} x_{\bar{p}}\right)^{-1}$ in (2.5) can be written as

$$
\left(x_{p} x_{\bar{p}}\right)^{-1}=x^{\sigma_{p}} .
$$


Now we take an additive subgroup $\Gamma$ of $\mathbb{F}^{2 \iota_{7}}$ such that

$$
\alpha_{\bar{I}_{5,6} \cup J_{7}}=0 \text { for } \alpha \in \Gamma \text {, }
$$

(this condition is necessary since we require that $T_{\overline{5}}=T_{\overline{6}}=T_{7}=T_{\overline{7}}=d$ by (2.12), which means that $\partial_{p}^{*}=0$, i.e., we shall have $\alpha_{p}=0$ if $p \in \bar{I}_{5,6} \cup J_{7}$ for $\alpha \in \Gamma$ (cf. (2.2) and (2.3))), and we shall also require that

$$
\sigma_{p} \in \Gamma, \quad \varepsilon_{q} \in \Gamma, \quad \mathbb{F} \varepsilon_{r} \cap \Gamma \neq\{0\} \quad \text { for } \quad p \in I_{1,4}, q \in I_{5,6}, r \in J_{1,4},
$$

where the first condition is necessary since we require that $x^{\sigma_{p}}$ will appear as a factor in the Lie bracket (cf. (2.5) and (2.24), also see (2.36)), and where the last two conditions are called the distinguishable conditions among the derivations $\partial_{p}$ defined later in (2.33), which are necessary in order to guarantee the simplicity of the Hamiltonian Lie algebras (cf. [X2]).

Note that $\mathbb{N}^{2 \iota_{7}}$ is an additive semi-subgroup of $\mathbb{F}^{2 \iota_{7}}$. We take

$$
\mathcal{J}=\left\{\underline{i}=\left(i_{1}, i_{\overline{1}}, \cdots, i_{\iota 7}, i_{\overline{\iota_{7}}}\right) \in \mathbb{N}^{2 \iota_{7}} \mid \underline{i}_{J_{1} \cup \bar{I}_{2,3} \cup I_{5}}=0\right\}
$$

(cf. (2.10)), where the condition $\underline{i}_{J_{1} \cup \bar{I}_{2,3} \cup I_{5}}=0$ is necessary since $T_{1}=T_{\overline{1}}=T_{\overline{2}}=T_{\overline{3}}=T_{5}=g$ by (2.12), which means that $\partial_{t_{p}}=0$, i.e., we shall have $i_{p}=0$ if $p \in J_{1} \cup \bar{I}_{2,3} \cup I_{5}$ for $\underline{i} \in \mathcal{J}$ (cf. (2.4) and (2.6)).

Now we let $\mathcal{A}=\mathbb{F}[\Gamma \times \mathcal{J}]$ be the semigroup algebra with basis

$$
\left\{x^{\alpha, \underline{i}} \mid(\alpha, \underline{i}) \in \Gamma \times \mathcal{J}\right\}
$$

(cf. (2.9)), and the multiplication

$$
x^{\alpha, \underline{i}} \cdot x^{\beta, \underline{j}}=x^{\alpha+\beta, \underline{i}+\underline{j}} \quad \text { for } \quad(\alpha, \underline{i}),(\beta, \underline{j}) \in \Gamma \times \mathcal{J} .
$$

Then $\mathcal{A}$ forms a commutative associative algebra with $1=x^{0,0}$ as the identity element. Set

$$
\mathcal{A}_{\alpha}=\operatorname{span}\left\{x^{\alpha, \underline{i}} \mid \underline{i} \in \mathcal{J}\right\} \quad \text { for } \quad \alpha \in \Gamma \text {. }
$$

Then $\mathcal{A}$ is $\Gamma$-graded $\mathcal{A}=\oplus_{\alpha \in \Gamma} \mathcal{A}_{\alpha}$ (but in general $\mathcal{A}_{\alpha}$ is infinite dimensional). For convenience, we denote

$$
x^{\alpha}=x^{\alpha, 0}, \quad t^{\underline{i}}=x^{0, \underline{i}}, \quad t_{p}=t^{\varepsilon_{p}}, \quad \text { for } \quad \alpha \in \Gamma, \underline{i} \in \mathcal{J}, p \in J .
$$

In particular,

$$
t^{\underline{i}}=\prod_{p \in J} t_{p}^{i_{p}}, \quad x^{\alpha, \underline{i}}=x^{\alpha} t^{\underline{i}}, \quad \text { for } \quad \alpha \in \Gamma, \underline{i} \in \mathcal{J},
$$

(cf. (2.9)). Define the derivations $\left\{\partial_{p}, \partial_{p}^{*}, \partial_{t_{p}} \mid p \in J\right\}$ of $\mathcal{A}$ by

$$
\partial_{p}=\partial_{p}^{*}+\partial_{t_{p}} \quad \text { and } \quad \partial_{p}^{*}\left(x^{\alpha, \underline{i}}\right)=\alpha_{p} x^{\alpha, \underline{i}}, \quad \partial_{t_{p}}\left(x^{\alpha, \underline{i}}\right)=i_{p} x^{\alpha, \underline{i}-\varepsilon_{p}},
$$

for $p \in J,(\alpha, \underline{i}) \in \Gamma \times \mathcal{J}$, where we treat

$$
x^{\alpha, \underline{i}}=0 \quad \text { if } \quad(\alpha, \underline{i}) \notin \Gamma \times \mathcal{J} .
$$


In particular,

$$
\partial_{p}^{*}=0, \quad \partial_{t_{q}}=0 \quad \text { for } \quad p \in \bar{I}_{5,6} \cup J_{7}, \quad q \in J_{1} \cup \bar{I}_{2,3} \cup I_{5},
$$

by (2.25) and (2.27) (cf. (2.12)). We call the nonzero derivations $\partial_{p}^{*}$ grading operators, the nonzero derivations $\partial_{t_{q}}$ down-grading operators, and the derivations $\partial_{r}^{*}+\partial_{t_{r}}$ mixed operators if both $\partial_{r}^{*}$ and $\partial_{t_{r}}$ are not zero. Then the types of derivation pairs in the order of the groups $\left\{\left(\partial_{p}, \partial_{\bar{p}}\right) \mid p \in I_{i}\right\}$ for $i \in \overline{1,7}$ are shown as in (2.12).

Now we define the following Lie bracket on $\mathcal{A}$ :

$$
[u, v]=\sum_{p \in I} x^{\sigma_{p}}\left(\partial_{p}(u) \partial_{\bar{p}}(v)-\partial_{\bar{p}}(u) \partial_{p}(v)\right),
$$

for $u \in \mathcal{A}_{\alpha}, v \in \mathcal{A}_{\beta}$ (cf. (2.30), (2.5) and (2.24)), where $x^{\sigma_{p}}$ appears just as in (2.5) and (2.24). Then $(\mathcal{A},[\cdot, \cdot])$ forms a Hamiltonian Lie algebra, denoted by $\mathcal{H}(\underline{\ell}, \Gamma)$, and $(\mathcal{A}, \cdot,[\cdot, \cdot])$ forms a Poisson algebra. Then $\mathcal{H}(\underline{\ell}, \Gamma)$ is the normalized form of a class of in general nongraded Hamiltonian Lie algebra constructed in [X2]. From this definition, one sees that the classical Hamiltonian Lie algebra $\mathcal{H}(\ell)$ is simply the Lie algebra $\mathcal{H}\left(\underline{\ell^{\prime}}, 0\right)$ with $\underline{\ell^{\prime}}=(0, \cdots, 0, \ell)$, and the Hamiltonian Lie algebras $\overline{\mathcal{H}}(\ell)$ and $\widehat{\mathcal{H}}(\ell)$ are respectively $\mathcal{H}\left(\underline{\ell^{\prime \prime}}, \mathbb{Z}^{\ell}\right)$ and $\mathcal{H}\left(\underline{\ell^{\prime \prime \prime}}, \mathbb{Z}^{\ell}\right)$, where $\underline{\ell^{\prime \prime}}=(\ell, 0, \cdots, 0)$, and $\underline{\ell^{\prime \prime \prime}}=(0,0,0, \ell, 0,0,0)$ (cf. (2.12) and the statement after it). The Hamiltonian Lie algebras considered in [OZ] in case $\phi_{0}=0$ are the cases of the Hamiltonian Lie algebras $[\mathcal{H}(\underline{\ell}, \Gamma), \mathcal{H}(\underline{\ell}, \Gamma)] / \mathbb{F}$ with $\underline{\ell}=(\ell, 0, \cdots, 0)$.

The Hamiltonian Lie algebras $\mathcal{H}(\underline{\ell}, \Gamma)$ can also be viewed as generalizations of the Lie algebras in $[\mathrm{DZ}, \mathrm{X} 1, \mathrm{Zh}]$ in the sense that they have some common features stated in Lemma 3.4 .

The following theorem was proved in [X2].

Theorem 2.1. The Lie algebra $\mathcal{H}(\underline{\ell}, \Gamma)$ is central simple, i.e., $[\mathcal{H}(\underline{\ell}, \Gamma), \mathcal{H}(\underline{\ell}, \Gamma)] / \mathbb{F}$ (the derived algebra modulo its center) is simple.

\section{Isomorphism Classes}

In this section, we shall determine the isomorphism classes of the Hamiltonian Lie algebras of the form $\mathcal{H}=\mathcal{H}(\underline{\ell}, \Gamma)$. We assume that $\mathbb{F}$ is an algebraically closed field.

By (2.25), (2.27) and (2.35), we can rewrite (2.36) in the following more explicit form:

$$
\begin{aligned}
{\left[x^{\alpha, \underline{i}}, x^{\beta, \underline{j}}\right]=} & \sum_{p \in I_{1,4}}\left(\alpha_{p} \beta_{\bar{p}}-\alpha_{\bar{p}} \beta_{p}\right) x^{\sigma_{p}+\alpha+\beta, \underline{i}+\underline{j}}+\sum_{p \in I_{5,6}}\left(\alpha_{p} j_{\bar{p}}-i_{\bar{p}} \beta_{p}\right) x^{\sigma_{p}+\alpha+\beta, \underline{i}+\underline{j}-\varepsilon_{\bar{p}}} \\
& +\sum_{p \in I_{2,4}}\left(i_{p} \beta_{\bar{p}}-j_{p} \alpha_{\bar{p}}\right) x^{\sigma_{p}+\alpha+\beta, \underline{i}+\underline{j}-\varepsilon_{p}} \\
& +\sum_{p \in I_{4} \cup I_{6,7}}\left(i_{p} j_{\bar{p}}-i_{\bar{p}} j_{p}\right) x^{\sigma_{p}+\alpha+\beta, \underline{\underline{i}}+\underline{j}-\varepsilon_{p}-\varepsilon_{\bar{p}}}
\end{aligned}
$$

for $(\alpha, \underline{i}),(\beta, \underline{j}) \in \Gamma \times \mathcal{J}$, where the first summand over $p \in I_{1,4}$ corresponds to the fact that $T_{i} \neq d \neq T_{\bar{i}}$ for $i=1,2,3,4$ (cf. (2.12)). As for other summands in (3.1), they are also obvious by (2.12). In particular, we have

$$
\left[x^{\alpha}, x^{\beta}\right]=\sum_{p \in I_{1,4}}\left(\alpha_{p} \beta_{\bar{p}}-\alpha_{\bar{p}} \beta_{p}\right) x^{\sigma_{p}+\alpha+\beta}=\sum_{p \in I_{1,4}}\left|\begin{array}{c}
\alpha_{\{p, \bar{p}\}} \\
\beta_{\{p, \bar{p}\}}
\end{array}\right| x^{\sigma_{p}+\alpha+\beta} \quad \text { for } \quad \alpha, \beta \in \Gamma,
$$


(cf. (2.31) and (2.22)), where $\left|\begin{array}{c}\alpha_{\{p, \bar{p}\}} \\ \beta_{\{p, \bar{p}\}}\end{array}\right|=\left|\begin{array}{cc}\alpha_{p} & \alpha_{\bar{p}} \\ \beta_{p} & \beta_{\bar{p}}\end{array}\right|$ is a $2 \times 2$ determinant, and

$$
\left[x^{-\sigma_{p}}, x^{\beta, \underline{j}}\right]=\left\{\begin{array}{lll}
\left(\beta_{p}-\beta_{\bar{p}}\right) x^{\beta, \underline{j}} & \text { if } & p \in I_{1}, \\
-\beta_{\bar{p}} x^{\beta, \underline{j}} & \text { if } & p \in I_{2}, \\
\left(\beta_{p}-\beta_{\bar{p}}\right) x^{\beta, \underline{j}}+j_{p} x^{\beta, \underline{j}-\varepsilon_{p}} & \text { if } & p \in I_{3}, \\
\left(\beta_{p}-\beta_{\bar{p}}\right) x^{\beta, \underline{j}}+j_{p} x^{\beta, \underline{j}-\varepsilon_{p}}-j_{\bar{p}} x^{\beta, \underline{j}-\varepsilon_{\bar{p}}} & \text { if } & p \in I_{4},
\end{array}\right.
$$

and

$$
\left[t_{\bar{q}}, x^{\beta, \underline{j}}\right]=\left\{\begin{array}{lll}
-\beta_{q} x^{\beta, \underline{j}} & \text { if } & q \in I_{5}, \\
-\beta_{q} x^{\beta, \underline{j}}-j_{q} x^{\beta, \underline{j}-\varepsilon_{q}} & \text { if } & q \in I_{6} .
\end{array}\right.
$$

For any $\underline{i} \in \mathcal{J}$, we define the level of $\underline{i}$ to be

$$
|\underline{i}|=\sum_{p \in J} i_{p}
$$

For any $(\alpha, \underline{i}) \in \Gamma \times \mathcal{J}$, we define the support of $(\alpha, \underline{i})$ to be

$$
\operatorname{supp}(\alpha, \underline{i})=\left\{p \in J \mid \alpha_{p} \neq 0 \text { or } i_{p} \neq 0\right\}
$$

For any Lie algebra $\mathcal{L}$, we denote by $\mathcal{L}^{F}$ and by $\mathcal{L}^{N}$ the sets of ad-locally finite elements and of ad-locally nilpotent elements, of $\mathcal{L}$ respectively. Generally, to obtain the isomorphism theorem, the ordinary way is first to find the sets $\mathcal{H}^{F}$ and $\mathcal{H}^{N}$. However, in our case here, the determinations of the sets $\mathcal{H}^{F}$ and $\mathcal{H}^{N}$ seem to be un-achievable. Thus, we use a "sandwich" method to estimate them. To do this, we introduce the following three subsets of $\mathcal{H}$. Denote

$$
\begin{gathered}
H_{1}=\left\{x^{-\sigma_{p}}, t_{\bar{q}} \mid p \in I_{1,4}, q \in I_{5,6}\right\}, \\
H_{2}=\left\{x^{\alpha, \underline{i}} \mid \alpha_{J_{1,4}}=\underline{i}_{J_{1,4} \cup \bar{I}_{5,6}}=0, i_{p} i_{\bar{p}}=0 \text { for } p \in J_{7}\right\}, \\
H_{3}=\operatorname{span}\left\{x^{\alpha, \underline{i}} \mid \alpha_{J_{1,4}}=\underline{i}_{J_{1,4} \cup \bar{I}_{5,6}}=0\right\},
\end{gathered}
$$

(cf. (2.22). Then our first result is the following "sandwich" lemma.

\section{Lemma 3.1.}

$$
\begin{gathered}
H_{1} \cup H_{2} \subset \mathcal{H}^{F} \subset \operatorname{span}\left(H_{1} \cup H_{3}\right), \\
H_{2} \subset \mathcal{H}^{N} \subset H_{3} .
\end{gathered}
$$

Proof. By (3.3) and (3.4), we have $H_{1} \subset \mathcal{H}^{F}$. Suppose $x^{\alpha, \underline{i}} \in H_{2}$. Then by (3.8),

$$
\operatorname{supp}(\alpha, \underline{i}) \subset I_{5,6} \cup J_{7}, \quad \text { and } \quad \bar{p} \notin \operatorname{supp}(\alpha, \underline{i}) \text { if } p \in \operatorname{supp}(\alpha, \underline{i}) .
$$

Let $x^{\beta, \underline{j}} \in \mathcal{H}$. By (3.1) and (3.12), we see

$$
\begin{aligned}
{\left[x^{\alpha, \underline{i}}, x^{\beta, \underline{j}}\right]=} & 0 \text { or a linear combination of the elements } x^{\gamma, \underline{k}} \text { such that } \\
& \text { there exists at least a } p \in\left(\bar{I}_{5,6} \cup J_{7}\right) \backslash \operatorname{supp}(\alpha, \underline{i}) \text { with } k_{p}<j_{p} .
\end{aligned}
$$

Thus if we set

$$
m=1+\sum_{p \in\left(\bar{I}_{5,6} \cup J_{7}\right) \backslash \operatorname{supp}(\alpha, \underline{i})} j_{p}
$$


then $\operatorname{ad}_{x^{\alpha, i}, \underline{i}}^{m}\left(x^{\beta, \underline{j}}\right)=0$. This proves $H_{2} \subset \mathcal{H}^{N} \subset \mathcal{H}^{F}$.

Suppose $u \notin \operatorname{span}\left(H_{1} \cup H_{3}\right)$. Write

$$
\begin{gathered}
u=\sum_{(\alpha, \underline{i}) \in S_{0}} c_{\alpha, \underline{i}} x^{\alpha, \underline{i}}, \quad \text { where } \\
S_{0}=\left\{(\alpha, \underline{i}) \in \Gamma \times \mathcal{J} \mid c_{\alpha, \underline{i}} \neq 0\right\} \quad \text { is a finite set. }
\end{gathered}
$$

Then by (3.7) and (3.9), there exist $(\gamma, \underline{k}) \in S_{0}$ and $p \in I_{1,6}$ such that at least one of $p$ and $\bar{p}$ is in $\operatorname{supp}(\gamma, \underline{k})$, mainly,

$$
\left(\gamma_{p}, \gamma_{\bar{p}}, k_{p}, k_{\bar{p}}\right) \neq 0
$$

and such that

$$
\begin{aligned}
& (\gamma, \underline{k}) \neq\left(-\sigma_{p}, 0\right) \quad \text { if } \quad p \in I_{1,4}, \quad \text { and } \\
& (\gamma, \underline{k}) \neq\left(0, \varepsilon_{\bar{p}}\right), \quad k_{\bar{p}} \neq 0 \quad \text { if } \quad p \in I_{5,6} .
\end{aligned}
$$

We prove that $u$ is not $a d$-locally finite. To do this, we choose a total order on $\Gamma$ compatible with group structure of $\Gamma$ and define the total order on $\Gamma \times \mathcal{J}$ by the lexicographical order, such that the maximal element $(\gamma, \underline{k})$ of $S_{0}$ satisfies (3.17)-(3.19) for some $p \in I_{1,6}$, and that $\sigma_{p}>\sigma_{q}$ for all $q \neq p$. This is possible because the set of all nonzero $\sigma_{q}$ is $\mathbb{F}$-linear independent. To see how it works, say, $p \in I_{1}$ and $\left(\gamma_{p}, \gamma_{\bar{p}}\right) \neq 0$ (the proof for other cases is similar). Choose $\beta=b \varepsilon_{\bar{p}} \in \Gamma$ for some $b \in \mathbb{F} \backslash\{0\}$ (cf. (2.26)) such that

$$
\gamma_{p} b+m\left(\gamma_{\bar{p}}-\gamma_{p}\right) \neq 0 \quad \text { for all } \quad m \in \mathbb{N} .
$$

Then for $n \in \mathbb{N}$, the "highest" term of $\operatorname{ad}_{u}^{n}\left(x^{\beta}\right)$ is $x^{\beta+n \gamma+n \sigma_{p}, n \underline{k}}$ with the coefficient

$$
\prod_{m=0}^{n-1}\left(\gamma_{p}\left(\beta_{\bar{p}}+m \gamma_{\bar{p}}-m\right)-\gamma_{\bar{p}}\left(m \gamma_{p}-m\right)\right)=\prod_{m=0}^{n-1}\left(\gamma_{p} b+m\left(\gamma_{\bar{p}}-\gamma_{p}\right) \neq 0 .\right.
$$

Thus by (3.18), the set $\left\{\operatorname{ad}_{u}^{n}\left(x^{\beta}\right) \mid n \in \mathbb{N}\right\}$ is linearly independent, which implies

$$
\operatorname{dim}\left(\operatorname{span}\left\{\operatorname{ad}_{u}^{n}\left(x^{\beta}\right) \mid n \in \mathbb{N}\right\}\right)=\infty .
$$

Thus $u \notin \mathcal{H}^{F}$. This proves $\mathcal{H}^{F} \subset \operatorname{span}\left(H_{1} \cup H_{3}\right)$. Similarly, $\mathcal{H}^{N} \subset H_{3}$.

For any subset $X \subset \mathcal{H}$, we denote by $E(X)$ the set of the zero vector and the common eigenvectors in $\mathcal{H}$ for $\operatorname{ad}_{X}$, mainly

$$
E(X)=\{u \in \mathcal{H} \mid[X, u] \subset \mathbb{F} u\} .
$$

Next, we shall determine $E\left(\mathcal{H}^{F}\right)$. To this end, we need to find the eigenvalues for elements of $\operatorname{ad}_{H_{1}}$. So we define a map $\pi: \Gamma \rightarrow \mathbb{F}^{\iota 6}$ by

$$
\begin{aligned}
& \pi(\alpha)=\mu=\left(\mu_{1}, \cdots, \mu_{\iota_{6}}\right), \quad \text { with } \\
& \mu_{p}= \begin{cases}\alpha_{p}-\alpha_{\bar{p}} & \text { if } p \in I_{1} \cup I_{3,4}, \\
-\alpha_{\bar{p}} & \text { if } p \in I_{2}, \\
-\alpha_{p} & \text { if } p \in I_{5,6},\end{cases}
\end{aligned}
$$


(cf. (3.3) and (3.4)). We define

$$
\begin{gathered}
\mathcal{M}=\operatorname{span}\left\{x^{\alpha} \in \mathcal{H} \mid \alpha \in \Gamma\right\} \\
\mathcal{M}_{\mu}=\operatorname{span}\left\{x^{\alpha} \mid \pi(\alpha)=\mu\right\} \quad \text { for } \quad \mu \in \pi(\Gamma) .
\end{gathered}
$$

Then we have

\section{Lemma 3.2.}

$$
E\left(\mathcal{H}^{F}\right)=\bigcup_{\mu \in \pi(\Gamma)} \mathcal{M}_{\mu}
$$

thus $\mathcal{M}=\operatorname{span}\left(E\left(\mathcal{H}^{F}\right)\right)$.

Proof. By (3.10) and the definition (3.23), we have

$$
E\left(H_{1} \cup H_{2}\right) \supset E\left(\mathcal{H}^{F}\right) \supset E\left(\operatorname{span}\left(H_{1} \cup H_{3}\right)\right) .
$$

We want to prove

$$
E\left(H_{1} \cup H_{2}\right) \subset \bigcup_{\mu \in \pi(\Gamma)} \mathcal{M}_{\mu} \subset E\left(\operatorname{span}\left(H_{1} \cup H_{3}\right)\right) .
$$

Let $\mu \in \pi(\Gamma)$. By (3.3), (3.4), (3.7)-(3.9) and (3.24)-(3.27), elements in $\mathcal{M}_{\mu}$ are common eigenvectors for $\operatorname{ad}_{H_{1}}$, and $\operatorname{ad}_{H_{3}}$ acts trivially on $\mathcal{M}_{\mu}$. Since elements in $H_{1}$ commute with each other, elements in $\mathcal{M}_{\mu}$ are common eigenvectors for $\operatorname{ad}_{\operatorname{span}\left(H_{1} \cup H_{3}\right)}$. That is,

$$
\bigcup_{\mu \in \pi(\Gamma)} \mathcal{M}_{\mu} \subset E\left(\operatorname{span}\left(H_{1} \cup H_{3}\right)\right) .
$$

Suppose

$$
u=\sum_{(\alpha, \underline{i}) \in S_{0}} c_{\alpha, \underline{\underline{x}}} x^{\alpha, \underline{i}} \in \mathcal{H}, \quad \text { where } \quad S_{0}=\left\{(\alpha, \underline{i}) \in \Gamma \times \mathcal{J} \mid c_{\alpha, \underline{i}} \neq 0\right\}
$$

is a common eigenvector for $\operatorname{ad}_{H_{1} \cup H_{2}}$. Since $\operatorname{ad}_{H_{2}}$ is locally nilpotent, $\operatorname{ad}_{H_{2}}$ must act trivially on $u$. If $(\alpha, \underline{i}) \in S_{0}$ with $i_{p} \neq 0$ for some $p \in \bar{I}_{5,6} \cup J_{7}$, then we can choose $v \in H_{2}$ :

$$
v=\left\{\begin{array}{lll}
x^{\varepsilon} \bar{p} & \text { if } & p \in \bar{I}_{5,6}, \\
t_{\bar{p}} & \text { if } & p \in J_{7}
\end{array}\right.
$$

such that $\left[v, x^{\alpha, \underline{i}}\right] \neq 0$ by $(3.1)$ and thus $[v, u] \neq 0$, contradicting the fact that $\operatorname{ad}_{H_{2}}$ acts trivially on $u$. Thus $\underline{i}_{\bar{I}_{5,6} \cup J_{7}}=0$. Similarly, since $u$ is a common eigenvector for $\operatorname{ad}_{H_{1}}$, we $\operatorname{must}$ have $\underline{i}_{I_{2,3} \cup J_{4}}=0$ (and thus $\underline{i}=0$ ) and $\pi(\alpha)=\mu$ for some $\mu$ if $(\alpha, \underline{i}) \in S_{0}$. This shows that $u \in \mathcal{M}_{\mu}$. This together with (3.31) proves (3.30). Now (3.29) and (3.30) show that all these sets are equal, i.e., we have (3.28).

Next we shall determine the sets $\mathcal{M}^{F}$ and $\mathcal{M}^{N}$. Recall that the Lie bracket in $\mathcal{M}$ has the simple form (3.2).

\section{Lemma 3.3.}

$$
\begin{gathered}
\mathcal{M}^{F}=\operatorname{span}\left\{x^{-\sigma_{p}}, x^{\alpha} \mid p \in I_{1,4}, \alpha_{J_{1,4}}=0\right\}, \\
\mathcal{M}^{N}=\operatorname{span}\left\{x^{\alpha} \mid \alpha_{J_{1,4}}=0\right\} .
\end{gathered}
$$


Proof. We shall prove (3.34) as the proof (3.35) is similar. It is straightforward to verify that by (3.2) elements in the right-hand side of (3.34) commute with each other and they are $a d$-locally finite on $\mathcal{M}$. Thus the right-hand side of (3.34) is contained in $\mathcal{M}^{F}$. Conversely, suppose $u \in \mathcal{M}$ is not in the right-hand side of (3.34). Then we can write $u$ as in (3.15), where now

$$
S_{0}=\left\{(\alpha, \underline{i}) \in \Gamma \times \mathcal{J} \mid \underline{i}=0, c_{\alpha, \underline{i}} \neq 0\right\} \quad \text { is a finite set. }
$$

Thus we still have (3.17)-(3.19), and the same arguments after (3.19) show that $u$ is not ad-locally finite on $\mathcal{M}$.

Now we shall study some important features of the Lie algebra $\mathcal{M}$, which is crucial in the proof of the isomorphism theorem.

Lemma 3.4. (1) Assume that $\iota_{4} \neq 0$. For $\mu \in \pi(\Gamma)$, regarding $\mathcal{M}_{\mu}$ as an $\mathcal{M}_{0}$-module via the adjoint action, we have (i) if $\mu_{I_{1,4}}=0$, then the action of $\mathcal{M}_{0}$ on $\mathcal{M}_{\mu}$ is trivial and (ii) if $\mu_{I_{1,4}} \neq 0$, then $\mathcal{M}_{\mu}$ is a cyclic $\mathcal{M}_{0}$-module, the nonzero multiplicative scalars of $x^{\alpha}$ for all $\alpha \in \Gamma$ with $\pi(\alpha)=\mu$, are the only generators.

(2) Assume that $\iota_{4}=0$ and $\iota_{6} \neq 0$. Then $\left(\cup_{\alpha \in \Gamma} \mathbb{F} x^{\alpha}\right) \backslash\{0\}$ are the set of the common eigenvectors of $\mathcal{H}^{F}$ in $\mathcal{M}$.

Proof. (1) Assume that $\iota_{4} \neq 0$. From (3.2) and the definition of $\pi$ in (3.24), we see that $x^{\alpha}$ commutes with $x^{\beta}$ if $\pi(\alpha)=0$ and $(\pi(\beta))_{I_{1,4}}=0$. Thus if $\mu_{I_{1,4}}=0$, the adjoint action of $\mathcal{M}_{0}$ on $\mathcal{M}_{\mu}$ is trivial. Assume

$$
\begin{aligned}
& u=\sum_{\beta \in S_{0}} c_{\beta} x^{\beta} \in \mathcal{M}_{\mu} \text { with } \quad \mu_{I_{1,4}} \neq 0, \text { where } \\
& S_{0}=\left\{\beta \in \Gamma \mid \pi(\beta)=\mu, c_{\beta} \neq 0\right\} \quad \text { is a finite set. }
\end{aligned}
$$

By (3.2), one has

$$
\left[x^{\alpha}, u\right]=-\sum_{p \in I_{1,4}} \alpha_{p} \mu_{p} x^{\sigma_{p}+\alpha} \cdot u \quad \text { if } \quad \pi(\alpha)=0 .
$$

Thus the subspace

$$
U=\operatorname{span}\left\{x^{\sigma_{p}+\alpha} \cdot u=\sum_{\beta \in S_{0}} c_{\beta} x^{\sigma_{p}+\alpha+\beta} \mid \alpha \in \operatorname{ker}_{\pi}, p \in I_{1,4}\right\},
$$

is a $\mathcal{M}_{0}$-submodule of $\mathcal{M}_{\mu}$. Let $\langle u\rangle$ denote the cyclic submodule of $\mathcal{M}_{\mu}$ generated by $u$. Then $\langle u\rangle \subset U$. If the size $\left|S_{0}\right|$ of $S_{0}$ is $\geq 2$, then $U$ in (3.40) is a proper submodule of $\mathcal{M}_{\mu}$ and so $u$ is not a generator of $\mathcal{M}_{\mu}$.

Now assume that $S_{0}$ is a singleton $\{\beta\}$ with $\pi(\beta)=\mu$. Suppose $\mu_{p} \neq 0$ for some $p \in I_{1,4}$. For any $k \neq 1$, by $(3.25), k \sigma_{p} \in \operatorname{ker}_{\pi}$, thus

$$
x^{\beta+k \sigma_{p}}=-\left((k-1) \mu_{p}\right)^{-1}\left[x^{(k-1) \sigma_{p}}, x^{\beta}\right] \in\langle u\rangle .
$$

For any $\alpha \in \operatorname{ker}_{\pi}$, by (3.25), $\alpha-(k+1) \sigma_{p} \in \operatorname{ker}_{\pi}$. Thus by (3.2), (3.25) and (3.41), noting that $\beta_{\bar{q}}=\beta_{q}-\mu_{q}$ for $q \in I_{1,4}$, it is straightforward to compute that

$$
k \mu_{p} x^{\alpha+\beta}+\sum_{q \in I_{1,4}}\left(\delta_{p, q}-\alpha_{q}\right) \mu_{q} x^{\alpha+\beta-\sigma_{p}+\sigma_{q}}=\left[x^{\alpha-(k+1) \sigma_{p}}, x^{\beta+k \sigma_{p}}\right] \in\langle u\rangle .
$$


This shows that $x^{\alpha+\beta} \in\langle u\rangle$ for all $\alpha \in \operatorname{ker}_{\pi_{1}}$, but $\mathcal{M}_{\mu}$ is spanned by such elements. Thus $u$ is a generator of $\mathcal{M}_{\mu}$.

(2) is obtained directly from (3.28).

Let $\mathcal{H}\left(\underline{\ell}^{\prime}, \Gamma^{\prime}\right)$ be another Hamiltonian Lie algebra defined in last section. We shall add a prime on all the constructional ingredients related to $\mathcal{H}\left(\underline{\ell}^{\prime}, \Gamma^{\prime}\right)$; for instance, $\mathcal{H}^{\prime}, \mathcal{J}^{\prime}, \sigma_{i}^{\prime}, \ell_{i}^{\prime}, \iota_{i}^{\prime}$, etc.

To state our isomorphism theorem, denote by $M_{m \times n}$ the space of $m \times n$ matrices with entries in $\mathbb{F}$ and by $G L_{m}$ the group of $m \times m$ invertible matrices with entries in $\mathbb{F}$.

Definition 3.5. Let $\Gamma, \Gamma^{\prime}$ be two additive subgroups of $\mathbb{F}^{2{ }^{27}}$ satisfying (2.25) and (2.26). A group isomorphism $\tau: \alpha \mapsto \alpha^{*}$ from $\Gamma \rightarrow \Gamma^{\prime}$ is called preserving if $\tau$ has the following form: there exists a permutation $\nu: p \mapsto p^{*}$ on the index set $I_{1,4}$, which maps $I_{k} \rightarrow I_{k}$ for $k=1,2,3,4$, such that

$$
\alpha_{\left\{p^{*}, \overline{p^{*}}\right\}}^{*}=\alpha_{\{p, \bar{p}\}} A_{p} \quad \text { for } \quad p \in I_{1,4},
$$

(cf. (2.22)), where $A_{p} \in G L_{2}$ and the multiplication in the right-hand side of (3.43) is the vector-matrix multiplication, and

$$
A_{p}=\left(\begin{array}{cc}
a_{p}+b_{p} & a_{p} \\
1-a_{p}-b_{p} & 1-a_{p}
\end{array}\right) \text { or }\left(\begin{array}{cc}
1 & 0 \\
a_{p} & b_{p}
\end{array}\right) \text { or }\left(\begin{array}{cc}
b_{p} & 0 \\
1-b_{p} & 1
\end{array}\right)
$$

if $p \in I_{1} \cup I_{4}$ or $I_{2}$ or $I_{3}$ respectively, for some $a_{p}, b_{p} \in \mathbb{F}$ with $b_{p} \neq 0$, and

$$
\begin{gathered}
\alpha_{I_{5}}^{*}=\left(\alpha_{I_{1}}-\alpha_{\bar{I}_{1}}\right) B_{1,5}-\alpha_{\bar{I}_{2}} B_{2,5}+\alpha_{I_{5}} B_{5,5}, \quad \text { where } \\
B_{1,5} \in M_{\ell_{1} \times \ell_{5}}, \quad B_{2,5} \in M_{\ell_{2} \times \ell_{5}}, \quad B_{5,5} \in G L_{\ell_{5}},
\end{gathered}
$$

and

$$
\begin{aligned}
& \alpha_{I_{6}}^{*}=\left(\alpha_{I_{1}}-\alpha_{\bar{I}_{1}}\right) B_{1,6}-\alpha_{\bar{I}_{2}} B_{2,6}+\left(\alpha_{I_{3,4}}-\alpha_{\bar{I}_{3,4}}\right) B_{3,6}+\alpha_{I_{5}} B_{5,6}+\alpha_{I_{6}} B_{6,6}, \quad \text { where } \\
& B_{1,6} \in M_{\ell_{1} \times \ell_{6}}, \quad B_{2,6} \in M_{\ell_{2} \times \ell_{6}}, \quad B_{3,6} \in M_{\left(\ell_{3}+\ell_{4}\right) \times \ell_{6}}, \quad B_{5,6} \in M_{\ell_{5} \times \ell_{6}}, \quad B_{6,6} \in G L_{\ell_{6}} .
\end{aligned}
$$

Note that the above uniquely determine the isomorphism by (2.25). Let us explain the above definition. First we introduce the following notations. For any $m \times n$ matrix $A=\left(a_{p, q}\right)$, we denote by $\widetilde{A}=\left(\widetilde{a}_{p, q}\right)$ (resp. $\left.\widehat{A}=\left(\widehat{a}_{p, q}\right)\right)$ the $2 m \times n$ matrix such that the odd rows of $\widetilde{A}$ (resp. $\widehat{A}$ ) forms the matrix $A$ (resp. the $m \times n$ zero matrix) and the even rows of $\widetilde{A}$ (resp. $\widehat{A}$ ) forms the matrix $-A$, i.e.,

$$
\widetilde{a}_{2 p-1, q}=-\widetilde{a}_{2 p, q}=a_{p, q}, \quad \widehat{a}_{2 p-1, q}=0, \widehat{a}_{2 p, q}=-a_{p, q} \quad \text { for } \quad p \in \overline{1, m}, q \in \overline{1, n} .
$$

A preserving isomorphism $\tau$ can be decomposed into the composition of two isomorphisms $\tau=\tau_{\nu} \cdot \tau_{0}$ such that $\tau_{\nu}$ only involves the permutation $\nu$, i.e., in (3.43)-(3.48), all $A_{p}$ and $B_{i, i}$ are identity matrices and all $B_{i, j}$ are zero matrices for $i \neq j$; and $\tau_{0}$ only involves matrices, i.e., $\nu=\mathbf{1}_{I_{1,4}}$ in (3.43). Furthermore, $\tau_{0}$ can be decomposed into $\tau_{0}=\tau_{1} \cdot \tau_{2}$ such that $\tau_{1}, \tau_{2}$ have the following forms

$$
\tau_{1}: \quad\left(\alpha_{J_{1,4}}^{*}, \alpha_{I_{5,6}}^{*}\right)=\left(\alpha_{J_{1,4}}, \alpha_{I_{5,6}}\right) A, \quad \text { where }
$$




$$
A=\operatorname{diag}\left(A_{1}, \cdots, A_{\iota_{4}}, B_{5,5}, B_{6,6}\right),
$$

and

$$
\tau_{2}: \quad\left(\alpha_{J_{1,4}}^{*}, \alpha_{I_{5,6}}^{*}\right)=\left(\alpha_{J_{1,4}}, \alpha_{I_{5,6}}\right) C, \quad C=\mathbf{1}_{2 \iota_{4}+\ell_{5}+\ell_{6}}+D,
$$

where in general $\mathbf{1}_{m}$ denotes the $m \times m$ identity matrix, and where $D$ has the form

$$
D=\left(0, D_{5}, D_{6}\right), \quad D_{5}=\left(\begin{array}{c}
\widetilde{B}_{1,5} \\
\widehat{B}_{2,5} \\
0
\end{array}\right), \quad D_{6}=\left(\begin{array}{c}
\widetilde{B}_{1,6} \\
\widehat{B}_{2,6} \\
B_{3,6} \\
B_{5,6} \\
0
\end{array}\right),
$$

where 0 denotes some proper zero matrices whose orders are clear from the context.

Now we can state the main result of this paper.

Theorem 3.6. $\theta: \mathcal{H}(\underline{\ell}, \Gamma) \cong \mathcal{H}\left(\underline{\ell}^{\prime}, \Gamma^{\prime}\right)$ if and only if $\underline{\ell}=\underline{\ell}^{\prime}$ and there exists a preserving isomorphism $\tau: \Gamma \cong \Gamma^{\prime}$.

Theorem 3.7 (Main Theorem). Two Hamiltonian Lie algebras are isomorphic if and only if their corresponding Poisson algebras are isomorphic.

Proof. By Theorem 3.6 and by [SX], the condition for two Hamiltonian Lie algebras being isomorphic is the same as the condition for the corresponding two Poisson algebras being isomorphic.

Proof of Theorem 3.6. " $\Leftarrow$ ": Suppose $\underline{\ell}=\underline{\ell}^{\prime}$ and $\tau: \Gamma \rightarrow \Gamma^{\prime}$ is a preserving isomorphism. By the explanation above, $\tau$ can be written as $\tau=\tau_{\nu} \cdot \tau_{1} \cdot \tau_{2}$, thus it suffices to consider the following 3 cases.

Case a: First assume that $\tau=\tau_{\nu}$ is determined by permutation $\nu$.

For any $\underline{i} \in \mathcal{J}$, we define $\underline{i}^{*} \in \mathcal{J}$ which is obtained from $\underline{i}$ by permutation $\nu$. Then it is straightforward to verify that the linear map

$$
\theta_{\nu}: \mathcal{H} \rightarrow \mathcal{H}^{\prime} \text { such that } \theta_{\nu}\left(x^{\alpha, \underline{i}}\right)=x^{\alpha^{*}, \underline{i}^{*}},
$$

is a Lie algebra isomorphism.

Case b: Next assume that $\tau=\tau_{1}$ as in (3.50).

We shall define an isomorphism $\theta: \mathcal{H} \rightarrow \mathcal{H}^{\prime}$ as Poisson algebra isomorphism (then $\theta$ is clearly a Lie algebra isomorphism). By (1.1), it suffices to find the images of the generators $x^{\alpha}, t_{p}$ for $\alpha \in \Gamma, p \in I_{2,4} \cup I_{6,7} \cup \bar{I}_{4,7}$ (cf. (3.58) and (3.62)-(3.64) below) such that the following conditions hold (cf. [SX]):

$$
\theta\left(\left[x^{\alpha}, x^{\beta}\right]\right)=\left[\theta\left(x^{\alpha}\right), \theta\left(x^{\beta}\right)\right], \quad \theta\left(\left[t_{p}, x^{\beta}\right]\right)=\left[\theta\left(t_{p}\right), \theta\left(x^{\beta}\right)\right], \quad \theta\left(\left[t_{p}, t_{q}\right]\right)=\left[\theta\left(t_{p}\right), \theta\left(t_{q}\right)\right],
$$

for $\alpha, \beta \in \Gamma$ and $p, q \in I_{2,4} \cup I_{6,7} \cup \bar{I}_{4,7}$.

Let $\Delta=\sum_{p \in I_{1,4}} \mathbb{Z} \sigma_{p}$ be the subgroup of $\Gamma$ generated by $\left\{\sigma_{p} \mid p \in I_{1,4}\right\}$ and define $\chi: \Delta \rightarrow$ $\mathbb{F}^{\times}=\mathbb{F} \backslash\{0\}$ to be the character of $\Delta$ (i.e., the group homomorphism $\Delta \rightarrow \mathbb{F}^{\times}$) determined by

$$
\chi\left(\sigma_{p}\right)=b_{p} \text { for } \quad p \in I_{1,4},
$$


where $b_{p}$ are elements in $\mathbb{F}$ appearing as entries of matrices $A_{p}$ in (3.44). We prove that $\chi$ can be extended to a character $\chi: \Gamma \rightarrow \mathbb{F}^{\times}$as follows: Assume that $\Delta_{1} \supset \Delta$ is a maximal subgroup of $\Gamma$ such that $\chi$ can be extended to a character $\chi: \Delta_{1} \rightarrow \mathbb{F}^{\times}$. If $\Delta_{1} \neq \Gamma$, then we choose $\alpha \in \Gamma \backslash \Delta_{1}$ and extend $\chi$ to $\Delta_{2}=\mathbb{Z} \alpha+\Delta_{1} \rightarrow \mathbb{F}^{\times}$by defining

$$
\chi(m \alpha+\beta)= \begin{cases}\chi(\beta) & \text { if } \mathbb{Z} \alpha \cap \Delta_{1}=\{0\}, \\ a^{m} \chi(\beta) & \text { if } \mathbb{Z} \alpha \cap \Delta_{1}=\mathbb{Z} n \alpha,\end{cases}
$$

for $m \in \mathbb{Z}, \beta \in \Delta_{1}$, where $a$ is an $n$th root of $\chi(n \alpha)$ in the second case (recall that $\mathbb{F}$ is algebraically closed). This leads to a contradiction with the maximality of $\Delta_{1}$. Thus $\chi$ can be extended to a character $\chi: \Gamma \rightarrow \mathbb{F}^{\times}$.

Now we define the images of $x^{\alpha}$ to be

$$
\theta\left(x^{\alpha}\right)=\chi(\alpha) x^{\prime \alpha^{*}} \quad \text { for } \quad \alpha \in \Gamma
$$

(recall that we add prime on the constructional ingredients related to $\mathcal{H}^{\prime}$ ). Then by (3.2) we see that the first equation of (3.55) holds because (3.44) and (3.50) guarantees that $\sigma_{p}^{*}=\sigma_{p}^{\prime}$ and that the determinant of $A_{p}$ is $\left|A_{p}\right|=b_{p}=\chi\left(\sigma_{p}\right)$ and

$$
\chi(\alpha) \chi(\beta)\left|\begin{array}{c}
\alpha_{\{p, \bar{p}\}}^{*} \\
\beta_{\{p, \bar{p}\}}^{*}
\end{array}\right|=\chi(\alpha+\beta)\left|\begin{array}{c}
\alpha_{\{p, \bar{p}\}} \\
\beta_{\{p, \bar{p}\}}
\end{array}\right| \cdot\left|A_{p}\right|=\chi\left(\sigma_{p}+\alpha+\beta\right)\left|\begin{array}{c}
\alpha_{\{p, \bar{p}\}} \\
\beta_{\{p, \bar{p}\}}
\end{array}\right| .
$$

Next we shall find the image of $t_{p}$. To do this, we introduce a new notation: For any vector $s=\left(s_{1}, s_{\overline{1}}, s_{2}, s_{\overline{2}}, \cdots, s_{\iota_{7}}, s_{\bar{\iota}_{7}}\right)$ (with entries in $\mathbb{F}, \mathcal{H}$ or in $\mathcal{H}^{\prime}$ ), we denote

$$
\bar{s}=\left(-s_{\overline{1}}, s_{1},-s_{\overline{2}}, s_{2}, \cdots,-s_{\bar{\iota}_{7}}, s_{\iota_{7}}\right) .
$$

For a subset $K \subset J$, we denote by $\bar{s}_{K}$ the vector obtained from $\bar{s}$ by deleting $-s_{\bar{p}}, s_{q}$ for $\bar{p}, q \in J \backslash K$; for instance,

$$
\bar{s}_{\{\overline{1}, \overline{2}, 3,4, \overline{4}\}}=\left(-s_{\overline{1}},-s_{\overline{2}}, s_{3},-s_{\overline{4}}, s_{4}\right),
$$

(cf. (2.22)). We define

$$
\begin{gathered}
\theta\left(t_{p}\right)=s_{p} \text { for } p \in I_{2,4} \cup I_{6,7} \cup \bar{I}_{4,7}, \quad \text { where } \\
s_{p}=t_{p}^{\prime}, \quad s_{q}=b_{q} t_{q}^{\prime}, \quad\left(-s_{\bar{r}}, s_{r}\right)=b_{r}\left(-t_{\bar{r}}^{\prime}, t_{r}^{\prime}\right) A_{r}^{-1} \quad \text { for } \quad p \in I_{2}, q \in I_{3}, r \in I_{4}, \\
\bar{s}_{\bar{I}_{5,6}}=\bar{t}_{\bar{I}_{5,6}}^{\prime} \operatorname{diag}\left(B_{5,5}, B_{6,6}\right)^{-1}, \quad \bar{s}_{I_{6}}=\bar{t}_{I_{6}}^{\prime} B_{6,6}^{T}, \quad s_{J_{7}}=t_{J_{7}}^{\prime},
\end{gathered}
$$

where the up-index "T" stands for the transpose of a matrix. Then if $p \in I_{2,3}$, we have

$$
\left[\theta\left(t_{p}\right), \theta\left(x^{\alpha}\right)\right]=\chi(\alpha) b_{p} \alpha_{\bar{p}} x^{\prime \alpha^{*}+\sigma_{p}^{\prime}}=\theta\left(\alpha_{\bar{p}} x^{\alpha+\sigma_{p}}\right)=\theta\left(\left[t_{p}, x^{\alpha}\right]\right)
$$

because by (3.44) and (3.50), $\alpha_{\bar{p}}^{*}=b_{p} \alpha_{\bar{p}}$ if $p \in I_{2}$ and $\alpha_{\bar{p}}^{*}=\alpha_{\bar{p}}$ if $p \in I_{3}$. If $p \in I_{4}$, as $1 \times 2$ matrices with entries in $\mathcal{H}$, we have

$$
\left[\theta\left(\bar{t}_{\{p, \bar{p}\}}\right), \theta\left(x^{\alpha}\right)\right]=\chi(\alpha) b_{p} \alpha_{\{p, \bar{p}\}}^{*} A_{p}^{-1} x^{\prime \alpha^{*}+\sigma_{p}^{\prime}}=\chi\left(\alpha+\sigma_{p}\right) \alpha_{\{p, \bar{p}\}} x^{\prime \alpha^{*}+\sigma_{p}^{\prime}}=\theta\left(\left[\bar{t}_{\{p, \bar{p}\}}, x^{\alpha}\right]\right) .
$$


Furthermore, we have $\left[\left(\bar{t}_{\bar{I}_{5,6}}, \bar{t}_{I_{6} \cup J_{7}}\right), x^{\alpha}\right]=\left(\alpha_{I_{5,6}}, 0\right) x^{\alpha}$, and

$$
\alpha_{I_{5,6}}^{*}=\alpha_{I_{5,6}} \operatorname{diag}\left(B_{5,5}, B_{6,6}\right),
$$

by (3.50). From this and (3.64), we obtain

$$
\left[\left(\theta\left(\bar{t}_{\bar{I}_{5,6}}\right), \theta\left(\bar{t}_{I_{6} \cup J_{7}}\right)\right), \theta\left(x^{\alpha}\right)\right]=\theta\left(\left[\left(\bar{t}_{\bar{I}_{5,6}}, \bar{t}_{I_{6} \cup J_{7}}\right), x^{\alpha}\right]\right) .
$$

From this and (3.66), we obtain the second equation of (3.55).

To verify the last equation of (3.55), note that

$$
\left[\bar{t}_{I_{2,3} \cup J_{4} \cup I_{5} \cup J_{6,7}}^{T}, \bar{t}_{I_{2,3} \cup J_{4} \cup I_{5} \cup J_{6,7}}\right]=\operatorname{diag}\left(0, S_{\ell_{4}}^{\sigma}, 0, S_{\ell_{6}+\ell_{7}}\right),
$$

where

$$
S_{\ell_{4}}^{\sigma}=\operatorname{diag}\left(\left(\begin{array}{cc}
0 & x^{\sigma_{\iota_{3}+1}} \\
-x^{\sigma_{\iota_{3}+1}} & 0
\end{array}\right), \cdots,\left(\begin{array}{cc}
0 & x^{\sigma_{\iota_{4}}} \\
-x^{\sigma_{\iota_{4}}} & 0
\end{array}\right)\right),
$$

is a $2 \ell_{4} \times 2 \ell_{4}$ matrix with entries in $\mathcal{H}$, and where, in general

$$
S_{m}=\operatorname{diag}\left(\left(\begin{array}{cc}
0 & 1 \\
-1 & 0
\end{array}\right), \cdots,\left(\begin{array}{cc}
0 & 1 \\
-1 & 0
\end{array}\right)\right) \in G L_{2 m} .
$$

Using (3.69), (3.63) and (3.64), we can obtain

$$
\left[\theta\left(\bar{t}_{I_{2,3} \cup J_{4} \cup I_{5} \cup J_{6,7}}\right)^{T}, \theta\left(\bar{t}_{I_{2,3} \cup J_{4} \cup I_{5} \cup J_{6,7}}\right)\right]=\theta\left(\left[\bar{t}_{I_{2,3} \cup J_{4} \cup I_{5} \cup J_{6,7}}^{T} \bar{t}_{I_{2,3} \cup J_{4} \cup I_{5} \cup J_{6,7}}\right]\right) .
$$

For example, if $p \in I_{4}$, by (3.56), (3.58) and (3.63), we have

$$
\begin{aligned}
{\left[\theta\left(\bar{t}_{\{p, \bar{p}\}}\right)^{T}, \theta\left(\bar{t}_{\{p, \bar{p}\}}\right)\right] } & =b_{p}\left(A_{p}^{-1}\right)^{T}\left[\bar{t}_{\{p, \bar{p}\}}^{\prime T}, \bar{t}_{\{p, \bar{p}\}}^{\prime}\right] b_{p} A_{p}^{-1} \\
& =b_{p}\left(\begin{array}{cc}
0 & x^{\prime \sigma_{p}^{\prime}} \\
-x^{\prime \sigma_{p}^{\prime}} & 0
\end{array}\right)=\theta\left(\left[\bar{t}_{\{p, \bar{p}\}}^{T}, \bar{t}_{\{p, \bar{p}\}}\right]\right) .
\end{aligned}
$$

This proves the last equation of (3.55).

Case c: Assume that $\tau=\tau_{2}$ as in (3.52).

We define (3.58) with $\chi(\alpha)=1$ and we define (3.62) with

$$
\begin{gathered}
\bar{s}_{I_{2,3} \cup J_{4}}=\bar{t}_{I_{2,3} \cup J_{4}}^{\prime}+\bar{t}_{I_{6}}^{\prime} E_{1}, \\
\bar{s}_{\bar{I}_{5,6}}=\bar{t}_{\bar{I}_{5,6}^{\prime}}^{\prime} E_{2}+\bar{t}_{I_{6}}^{\prime} E_{3}+x^{\prime-\sigma^{\prime}} E_{4}, \quad \bar{s}_{I_{6} \cup J_{7}}=\bar{t}_{I_{6} \cup J_{7}}^{\prime},
\end{gathered}
$$

where $E_{1}, \ldots, E_{4}$ are some matrices to be determined in order that (3.55) holds and where $x^{\prime-\sigma^{\prime}}$ denotes the vector

$$
x^{\prime-\sigma^{\prime}}=\left(x^{\prime-\sigma_{1}^{\prime}}, \cdots, x^{\prime-\sigma_{\iota_{4}}^{\prime}}\right) .
$$

We shall not give the explicit forms of $E_{1}, \ldots, E_{4}$ here, but an interested reader can find the solutions by considering two special cases of (3.53): (1) $D_{5}=0$, (2) $D_{6}=0$ (the general case is the composition of the two special cases), or refer to [SX] (also, cf. the proof of necessity). 
" $\Rightarrow$ ": Assume that there exits a Hamiltonian Lie algebra isomorphism $\theta: \mathcal{H}(\underline{\ell}, \Gamma) \rightarrow$ $\mathcal{H}\left(\underline{\ell}^{\prime}, \Gamma^{\prime}\right)$.

First, we make the following conventions: If a subset of $\mathcal{H}$ is defined, then we take the definition of the corresponding subset of $\mathcal{H}^{\prime}$ for granted. If a property about $\mathcal{H}$ is given, the same property also holds for $\mathcal{H}^{\prime}$, without description.

Clearly, $\theta$ maps $\mathcal{H}^{F}, \mathcal{H}^{N}$ to $\mathcal{H}^{\prime F}, \mathcal{H}^{\prime N}$ respectively, thus also maps $\mathcal{M} \rightarrow \mathcal{M}^{\prime}$ by Lemma 3.2. By Lemma 3.3, we have $\operatorname{dim}\left(\mathcal{M}^{F} / \mathcal{M}^{N}\right)=\iota_{4}$. This shows that

$$
\iota_{4}=\iota_{4}^{\prime} .
$$

For simplicity, we assume that $\iota_{4} \neq 0$ (if $\iota_{4}=0$, using Lemma 3.4 (2), one sees that all statements or arguments below either work or do not apply to the case; if $\iota_{6}=0$, then one can go directly to Claim 8 below). Denote

$$
\Gamma_{1,4}=\left\{\alpha \in \Gamma \mid(\pi(\alpha))_{I_{1,4}}=0\right\},
$$

(cf. (3.24) and (2.22)). By Lemma 3.2, there exists a bijection $\tau_{1}: \pi(\Gamma) \rightarrow \pi\left(\Gamma^{\prime}\right)$ such that

$$
\theta\left(\mathcal{M}_{\mu}\right)=\mathcal{M}_{\tau_{1}(\mu)}^{\prime} \quad \text { for } \quad \mu \in \pi(\Gamma), \quad \text { and } \tau_{1}(0)=0 .
$$

From this and Lemma 3.4, there exists a bijection $\Gamma \backslash \Gamma_{1,4} \rightarrow \Gamma^{\prime} \backslash \Gamma_{1,4}^{\prime}$ which shall be denoted by $\tau: \alpha \mapsto \alpha^{*}$ such that

$$
\theta\left(x^{\alpha}\right)=c_{\alpha} x^{\prime \alpha^{*}} \quad \text { for } \quad \alpha \in \Gamma \backslash \Gamma_{1,4} \text { and some } c_{\alpha} \in \mathbb{F}^{\times} .
$$

We shall prove the necessity by establishing several claims.

Claim 1. There exists a bijection $I_{1,4} \rightarrow I_{1,4}^{\prime}$ denoted by $\nu: p \mapsto p^{*}$ such that

$$
\theta\left(x^{-\sigma_{p}}\right)=d_{p} x^{-\sigma_{p^{*}}^{\prime}} \text { for } p \in I_{1,4} \text { and some } d_{p} \in \mathbb{F}^{\times} .
$$

By (3.7)-(3.9) and Lemma 3.3, we have

$$
\left\{u \in \mathcal{M}^{F} \mid\left[u, H_{1} \cup H_{2}\right]=0\right\}=\operatorname{span}\left\{x^{-\sigma_{p}} \mid p \in I_{1,4}\right\}=\left\{u \in \mathcal{B}^{F} \mid\left[u, H_{1} \cup H_{3}\right]=0\right\} .
$$

Thus by Lemma 3.1,

$$
\left\{u \in \mathcal{M}^{F} \mid\left[u, \mathcal{H}^{F}\right]=0\right\}=\operatorname{span}\left\{x^{-\sigma_{p}} \mid p \in I_{1,4}\right\} .
$$

Let $p \in I_{1,4}$. Then by (3.83),

$$
\theta\left(x^{-\sigma_{p}}\right) \in \sum_{q \in I_{1,4}^{\prime}} \mathbb{F} x^{\prime-\sigma_{q}^{\prime}}
$$

Suppose

$$
\theta\left(x^{-\sigma_{p}}\right) \notin \bigcup_{q \in I_{1,4}^{\prime}} \mathbb{F} x^{\prime-\sigma_{q}^{\prime}} .
$$

By (2.26), there exists $a \in \mathbb{F}^{\times}$such that $a \varepsilon_{\bar{p}} \in \Gamma$. By (3.2), we have

$$
\left[x^{a \varepsilon_{\bar{p}}-\sigma_{p}}, x^{-a \varepsilon_{\bar{p}}-\sigma_{p}}\right]=2 a x^{-\sigma_{p}} .
$$


Note that $a \varepsilon_{\bar{p}}-\sigma_{p},-a \varepsilon_{\bar{p}}-\sigma_{p} \notin \Gamma_{1,4}$, by $(3.81)$,

$$
\theta\left(x^{a \varepsilon_{\bar{p}}-\sigma_{p}}\right) \in \mathbb{F} x^{\prime \alpha} \backslash\{0\}, \quad \theta\left(x^{-a \varepsilon_{\bar{p}}-\sigma_{p}}\right) \in \mathbb{F} x^{\prime \beta} \backslash\{0\} \quad \text { for some } \alpha, \beta \in \Gamma^{\prime} \backslash \Gamma_{1,4}^{\prime} .
$$

By (3.2), we have

$$
\left[x^{\prime \alpha}, x^{\prime \beta}\right]=\sum_{q \in I_{1,4}^{\prime}}\left(\alpha_{q} \beta_{\bar{q}}-\alpha_{\bar{q}} \beta_{q}\right) x^{\prime \sigma_{q}^{\prime}+\alpha+\beta} .
$$

By (3.84)-(3.86) and (3.88), there exist $q, r \in I_{1,4}^{\prime}$ with $q \neq r$ such that $\sigma_{q}^{\prime}+\alpha+\beta=-\sigma_{r}^{\prime}$. Thus

$$
\beta=-\alpha-\sigma_{q}^{\prime}-\sigma_{r}^{\prime}
$$

and (3.88) becomes

$$
\left[x^{\prime \alpha}, x^{\prime \beta}\right]=\left(\alpha_{q} \eta_{\bar{q}}^{\prime}+\alpha_{\bar{q}}\right) x^{\prime-\sigma_{r}^{\prime}}+\left(\alpha_{r} \eta_{\bar{r}}^{\prime}+\alpha_{\bar{r}}\right) x^{\prime-\sigma_{q}^{\prime}}
$$

where in general, for $q \in J_{1,4}$, we denote

$$
\eta_{q}= \begin{cases}1 & \text { if } q \in I_{1,4} \\ -1 & \text { if } q \in \bar{I}_{1} \cup I_{3,4} \\ 0 & \text { if } \quad q \in \bar{I}_{2}\end{cases}
$$

and we define $\eta_{q}^{\prime}$ similarly (then $\sigma_{q}^{\prime}=\varepsilon_{q}^{\prime}-\eta_{\bar{q}}^{\prime} \varepsilon_{\bar{q}}^{\prime}$, cf. (2.23)). By (3.85), both coefficients in (3.90) are nonzero. Since $2 a \varepsilon_{\bar{p}}-\sigma_{p} \in \Gamma \backslash \Gamma_{1,4}$, we have

$$
\theta\left(x^{2 a \varepsilon_{\bar{p}}-\sigma_{p}}\right) \in \mathbb{F} x^{\prime \gamma} \backslash\{0\} \text { for some } \gamma \in \Gamma^{\prime} \backslash \Gamma_{1,4}^{\prime}
$$

From $\left[x^{2 a \varepsilon_{\bar{p}}-\sigma_{p}}, x^{-a \varepsilon_{\bar{p}}-\sigma_{p}}\right] \in \mathbb{F} x^{a \varepsilon_{\bar{p}}-\sigma_{p}}$, it follows from (3.87) that

$$
\left[x^{\prime \gamma}, x^{\beta}\right] \in \mathbb{F} x^{\prime \alpha}
$$

Thus there exists $q^{\prime} \in I_{1,4}^{\prime}$ such that

$$
\gamma_{q^{\prime}} \beta_{\bar{q}^{\prime}}-\gamma_{\bar{q}^{\prime}} \beta_{q^{\prime}} \neq 0 \text { and } \sigma_{q^{\prime}}^{\prime}+\gamma+\beta=\alpha .
$$

Hence

$$
\gamma=\alpha-\beta-\sigma_{q^{\prime}}^{\prime}=2 \alpha+\sigma_{q}^{\prime}+\sigma_{r}^{\prime}-\sigma_{q^{\prime}}^{\prime},
$$

by (3.89). If $q \neq q^{\prime} \neq r$, we deduce from (3.89) and (3.95) that

$$
\begin{aligned}
{\left[x^{\prime \gamma}, x^{\prime \beta}\right]=} & \left(\alpha_{q} \eta_{\bar{q}}^{\prime}+\alpha_{\bar{q}}\right) x^{\prime \sigma_{q}^{\prime}+\gamma+\beta}+\left(\alpha_{r} \eta_{\bar{r}}^{\prime}+\alpha_{\bar{r}}\right) x^{\prime \sigma_{r}^{\prime}+\gamma+\beta} \\
& +\left(\gamma_{q^{\prime}} \beta_{\bar{q}^{\prime}}-\gamma_{\bar{q}^{\prime}} \beta_{q^{\prime}}\right) x^{\prime \sigma_{q^{\prime}}^{\prime}+\gamma+\beta} \notin \mathbb{F} x^{\prime \alpha},
\end{aligned}
$$

a contradiction with (3.93). Similarly, if $q^{\prime}=q$ or $q^{\prime}=r$, we can still deduce a contradiction from (3.89), (3.93) and (3.95). This proves the claim.

We extend $\nu$ to $\nu: J_{1,4} \rightarrow J_{1,4}^{\prime}$ such that $\nu(\bar{p})=\bar{p}^{*}$ for $p \in I_{1,4}$. For $p \in I_{1,4}$, by (2.26), we fix $e_{p} \in \mathbb{F}^{\times}$such that

$$
\lambda_{p}=e_{p} \varepsilon_{\bar{p}} \in \Gamma \backslash\{0\}
$$


Then $\lambda_{p} \notin \Gamma_{1,4}$. Denote $\lambda_{p}^{*}=\tau\left(\lambda_{p}\right)$ (cf. (3.80)). Write

$$
\lambda_{p}^{*}=\left(\lambda_{p, 1}^{*}, \lambda_{p, \overline{1}}^{*}, \cdots, \lambda_{p, \iota_{7}^{\prime}}^{*}, \lambda_{p, \tau_{7}^{\prime}}^{*}\right) \in \Gamma^{\prime} \subset \mathbb{F}^{2 \iota_{7}^{\prime}},
$$

(cf. (2.20)). For $p, q \in I_{1,4}$, applying $\theta$ to $\left[x^{\lambda_{p}}, x^{-\sigma_{q}}\right]=\delta_{p, q} e_{p} x^{\lambda_{p}}$, by (3.80) and (3.81), we obtain

$$
d_{q}\left(\eta_{\bar{q}^{*}}^{\prime} \lambda_{p, q^{*}}^{*}+\lambda_{p, \bar{q}^{*}}^{*}\right)=\delta_{p, q} e_{p} \quad \text { for } \quad p, q \in I_{1,4} .
$$

Let $p \neq q$. Applying $\theta$ to $0=\left[x^{\lambda_{p}}, x^{\lambda_{q}}\right]$ and using (3.99), we obtain

$$
0=\lambda_{p, q^{*}}^{*} \lambda_{q, \bar{q}^{*}}^{*}-\lambda_{p, \bar{q}^{*}}^{*} \lambda_{q, q^{*}}^{*}=\lambda_{p, q^{*}}^{*}\left(\lambda_{q, \bar{q}^{*}}^{*}+\eta_{\bar{q}^{*}}^{\prime} \lambda_{q, q^{*}}^{*}\right)=\lambda_{p, q^{*}}^{*} d_{q}^{-1} e_{q} .
$$

The above two equations imply

$$
\lambda_{p, q^{*}}^{*}=0 \quad \text { for } p \in I_{1,4}, q \in J_{1,4}, q \neq p, \bar{p} .
$$

Denote

$$
\Gamma_{p}=\left(\mathbb{F} \varepsilon_{p}+\mathbb{F} \varepsilon_{\bar{p}}\right) \cap \Gamma .
$$

Exactly to the proof of (3.101), we have

$$
\alpha_{q^{*}}^{*}=0 \quad \text { for } \quad \alpha \in \Gamma_{p} \backslash \Gamma_{1,4}, p, q \in J_{1,4}, q \neq p, \bar{p} .
$$

Claim 2. $\tau: \alpha \mapsto \alpha^{*}$ can be uniquely extended to a group isomorphism $\tau: \Gamma \rightarrow \Gamma^{\prime}$ such that $\sigma_{p}^{*}=\sigma_{p^{*}}^{\prime}$ for $p \in I_{1,4}$.

Noting that by (3.24), (3.25) and (3.78), $\alpha \notin \Gamma_{1,4}$ implies $\alpha+k \sigma_{1} \notin \Gamma_{1,4}$ for $k \in \mathbb{Z}$. For any $\alpha \in \Gamma, \beta \in \Gamma_{1}$ with $\alpha, \beta, \alpha+\beta \notin \Gamma_{1,4}$, we have (recall (3.91))

$$
\begin{aligned}
\left(\alpha_{1}\left(\beta_{\overline{1}}+\eta_{\overline{1}}\right)\right. & \left.-\alpha_{\overline{1}}\left(\beta_{1}-1\right)\right) c_{\alpha+\beta-\sigma_{1}} x^{\prime(\alpha+\beta)^{*}} \\
& =c_{\alpha} c_{\beta-\sigma_{1}}\left(\alpha_{1^{*}}^{*}\left(\beta-\sigma_{1}\right)_{\overline{1}^{*}}^{*}-\alpha_{\overline{1}^{*}}^{*}\left(\beta-\sigma_{1}\right)_{1^{*}}^{*}\right) x^{\prime \sigma_{1^{*}}^{\prime}+\alpha^{*}+\left(\beta-\sigma_{1}\right)^{*}},
\end{aligned}
$$

by applying $\theta$ to (3.2) and by (3.103). By comparing the power of $x^{\prime}$, this implies

$$
(\alpha+\beta)^{*}=\sigma_{1^{*}}^{\prime}+\alpha^{*}+\left(\beta-\sigma_{1}\right)^{*}
$$

if $\alpha, \beta$ satisfy

$$
\beta \in \Gamma_{1}, \quad \alpha, \beta, \alpha+\beta \in \Gamma \backslash \Gamma_{1,4}, \quad \text { and } \quad \alpha_{1}\left(\beta_{\overline{1}}+\eta_{\overline{1}}\right)-\alpha_{\overline{1}}\left(\beta_{1}-1\right) \neq 0 .
$$

Let $\alpha \in \Gamma \backslash \Gamma_{1,4}$. We prove by induction on $|k|$ that

$$
(k \alpha)^{*}-k \alpha^{*} \in \widetilde{\Gamma}_{1}^{\prime} \text {, where } \widetilde{\Gamma}_{1}^{\prime}=\left\{\beta \in \Gamma^{\prime} \mid \beta_{q}=0 \text { for } q \in J_{1,4}, q \neq 1^{*}, \overline{1}^{*}\right\} .
$$

Let $\gamma \in \Gamma$ such that $\gamma, \alpha+\gamma \notin \Gamma_{1,4}$. We have

$$
\sum_{p \in I_{1,4}}\left(\alpha_{p} \gamma_{\bar{p}}-\alpha_{\bar{p}} \gamma_{p}\right) c_{\sigma_{p}+\alpha+\gamma} x^{\prime\left(\sigma_{p}+\alpha+\gamma\right)^{*}}=c_{\alpha} c_{\gamma} \sum_{p \in I_{1,4}^{\prime}}\left(\alpha_{p^{*}}^{*} \gamma_{\bar{p}^{*}}^{*}-\alpha_{\bar{p}^{*}}^{*} \gamma_{p^{*}}^{*}\right) x^{\prime \sigma_{p^{*}}^{\prime}+\alpha^{*}+\gamma^{*}} .
$$

We inductively assume that (3.107) holds for $k$ (for instance, $k=1$ ). Let $\gamma=k \alpha+\beta$ for some suitable $\beta \in \Gamma_{1}$ such that condition (3.106) holds for all the involved pairs for which 
we need to make use of (3.105) in the following proof (when $\alpha, k$ are fixed, by (2.26), such $\beta$ exists), by (3.107) (note that we assume (3.107) holds for $k$ ), (3.105) and (3.103), we see that all terms in (3.108) vanish except the terms corresponding to $p=1$ in both sides. Thus we obtain

$$
\begin{aligned}
\sigma_{1^{*}}^{\prime}+((k+1) \alpha)^{*}+\beta^{*} & =\left(\sigma_{1}+(k+1) \alpha+\beta\right)^{*} \\
& =\sigma_{1^{*}}^{\prime}+\alpha^{*}+(k \alpha+\beta)^{*}=2 \sigma_{1^{*}}^{\prime}+\alpha^{*}+(k \alpha)^{*}+\left(\beta-\sigma_{1}\right)^{*},
\end{aligned}
$$

where the first and last equalities follow from (3.105) and the second follows from (3.108). From this we see that (3.107) holds for $k+1$. This proves (3.107). Now replacing $\alpha$ by $j \alpha$ (with $j \neq 0$ ) and $\beta$ by $k \alpha+\beta-\sigma_{1}$ in (3.108) (with suitable $\beta \in \Gamma_{1}$ ), since (3.107) holds, we have again that all terms in (3.108) vanish except the terms corresponding to $p=1$ in both sides. Thus we have similar formula as in (3.109):

$$
((j+k) \alpha+\beta)^{*}=2 \sigma_{1^{*}}^{\prime}+(j \alpha)^{*}+(k \alpha)^{*}+\left(\beta-2 \sigma_{1}\right)^{*} .
$$

From this we obtain

$$
(j \alpha)^{*}+(k \alpha)^{*}=\left(j^{\prime} \alpha\right)^{*}+\left(k^{\prime} \alpha\right)^{*} \text { if } j+k=j^{\prime}+k^{\prime}, j, k, j^{\prime}, k^{\prime} \neq 0 .
$$

From this we obtain

$$
(j \alpha)^{*}=j \alpha^{*} \quad \text { for } \quad \alpha \in \Gamma \backslash \Gamma_{1,4}, j \in \mathbb{Z} \backslash\{0\} .
$$

For some suitable $\beta \in \Gamma_{1}$, by (3.105), (3.110) and (3.112), we have

$$
\begin{aligned}
\sigma_{1^{*}}^{\prime}+\left(j \alpha+\sigma_{1}\right)^{*}+\left(\beta-2 \sigma_{1}\right)^{*} & =\left(\left(j \alpha+\sigma_{1}\right)+\left(\beta-\sigma_{1}\right)\right)^{*} \\
& =(j \alpha+\beta)^{*}=2 \sigma_{1^{*}}^{\prime}+j \alpha^{*}+\left(\beta-2 \sigma_{1}\right)^{*} .
\end{aligned}
$$

From this we obtain

$$
\left(j \alpha+\sigma_{1}\right)^{*}=j \alpha^{*}+\sigma_{1^{*}}^{\prime} \quad \text { for } \quad \alpha \in \Gamma \backslash \Gamma_{1,4}, j \in \mathbb{Z} \backslash\{0\} .
$$

Now take any $\alpha, \gamma \in \Gamma$ such that

$$
\alpha, \gamma, \alpha+\gamma \in \Gamma \backslash \Gamma_{1,4} \text { and } \alpha_{1} \gamma_{\overline{1}}-\alpha_{\overline{1}} \gamma_{1} \neq 0
$$

Using (3.114) in (3.108), by comparing the term $x^{\left(\sigma_{1}+\alpha+\gamma\right)^{*}}$ in both sides, we obtain

$$
\begin{aligned}
& (\alpha+\gamma)^{*}=\alpha^{*}+\gamma^{*}+\sum_{p \in I_{1,4}} k_{\alpha, \gamma}^{(p)}\left(\sigma_{p^{*}}^{\prime}-\sigma_{1^{*}}^{\prime}\right), \text { where } \\
& k_{\alpha, \gamma}^{(p)}=0,1 \text { such that } \sum_{p \in I_{1,4}} k_{\alpha, \gamma}^{(p)} \leq 1 .
\end{aligned}
$$

We claim that $(\alpha+\gamma)^{*}=\alpha^{*}+\gamma^{*}$ if the pairs $(\alpha, \gamma),(2 \alpha, 2 \gamma)$ satisfy (3.115). Assume that $k_{\alpha, \gamma}^{(q)}=1$ for some $q \in I_{1,4}$. Then we obtain

$$
\begin{aligned}
(2 \alpha)^{*}+(2 \gamma)^{*}+\sum_{p \in I_{1,4}} k_{2 \alpha, 2 \gamma}^{(p)}\left(\sigma_{p^{*}}^{\prime}-\sigma_{1^{*}}^{\prime}\right) & =(2 \alpha+2 \gamma)^{*}=(2(\alpha+\gamma))^{*}=2(\alpha+\gamma)^{*} \\
& =2\left(\alpha^{*}+\gamma^{*}+\sum_{p \in I_{1,4}} k_{\alpha, \gamma}^{(p)}\left(\sigma_{p^{*}}^{\prime}-\sigma_{1^{*}}^{\prime}\right)\right),
\end{aligned}
$$


from this we obtain $k_{2 \alpha, 2 \gamma}^{(q)}=2 k_{\alpha, \gamma}^{(q)}>1$, which is a contradiction to (3.116).

For any $\alpha, \beta, \alpha+\beta \in \Gamma \backslash \Gamma_{1,4}$, we can always choose $\gamma \in \Gamma \backslash \Gamma_{1,4}$ such that the pairs

$$
(\alpha+\beta, \gamma),(2 \alpha+2 \beta, 2 \gamma),(\alpha, \beta+\gamma),(2 \alpha, 2 \beta+2 \gamma),(\beta, \gamma),(2 \beta, 2 \gamma)
$$

satisfy (3.115). Hence

$$
(\alpha+\beta)^{*}+\gamma^{*}=(\alpha+\beta+\gamma)^{*}=\alpha^{*}+(\beta+\gamma)^{*}=\alpha^{*}+\beta^{*}+\gamma^{*},
$$

which shows

$$
(\alpha+\beta)^{*}=\alpha^{*}+\beta^{*} \text { for } \alpha, \beta, \alpha+\beta \in \Gamma \backslash \Gamma_{1,4} .
$$

This shows that $\tau$ can be uniquely extended to a group isomorphism $\tau: \Gamma \rightarrow \Gamma^{\prime}$ such that $\sigma_{1}^{*}=\sigma_{1^{*}}^{\prime}$ and so similarly $\sigma_{p}^{*}=\sigma_{p^{*}}^{\prime}$ for $p \in I_{1,4}$. This proves the claim.

Claim 3. We have $\nu\left(I_{i}\right)=I_{i}^{\prime}$ for $i=1,2,3,4$. In particular, $\left(\ell_{1}, \ell_{2}, \ell_{3}, \ell_{4}\right)=\left(\ell_{1}^{\prime}, \ell_{2}^{\prime}, \ell_{3}^{\prime}, \ell_{4}^{\prime}\right)$, $I_{i}=I_{i}^{\prime}$ for $i=1,2,3,4$, and $\sigma_{p}=\sigma_{p}^{\prime}, \eta_{p}=\eta_{p}^{\prime}$ for $p \in I_{1,4}$ (cf. (2.23) and (3.91)).

Note that $\operatorname{ad}_{x^{-\sigma_{p}}}$ is a semi-simple operator on $\mathcal{H}$ if and only if $p \in I_{1,2}$ (cf. (3.3)). Thus

$$
\nu\left(I_{1,2}\right)=I_{1,2}^{\prime}, \quad \text { and so } \nu\left(I_{3,4}\right)=I_{3,4}^{\prime} \text {. }
$$

Denote

$$
\begin{aligned}
& \mathcal{N}=\{u \in \mathcal{H} \mid[u, \mathcal{M}] \subset \mathcal{M}\} \\
&=\mathcal{M}+\operatorname{span}\left\{x^{\alpha, \underline{i}}\left|\alpha=\alpha_{I_{5,6}},\right| \underline{i} \mid=1 \text { or } \underline{i}=\underline{i}_{I_{6} \cup J_{7}}\right\}, \\
& \mathcal{N}_{0}= \mathcal{M}+\left\{u \in \mathcal{N} \mid\left[x^{-\sigma_{p}}, u\right]=0 \text { for } p \in I_{1,4}\right\} \\
&= \mathcal{M}+\operatorname{span}\left\{x^{\alpha, \varepsilon_{q}}, x^{\alpha, \underline{j}} \mid \alpha=\alpha_{I_{5,6}}, q \in \bar{I}_{5,6}, \underline{j}=\underline{j}_{I_{6} \cup J_{7}}\right\}, \\
& \mathcal{N}_{p}=\mathcal{M}+\operatorname{span}\left\{u \in \mathcal{N} \mid\left[x^{-\sigma_{p}}, u\right]=0\right\} \text { for } p \in I_{1,4} .
\end{aligned}
$$

Then $\mathcal{N}_{0}$ is a Lie algebra and $\mathcal{N}$ is an $\mathcal{N}_{0}$-module such that $\mathcal{N}_{p}$ is a submodule for $p \in I_{1,4}$. Note that the quotient module $\mathcal{N} / \mathcal{N}_{p}$ is zero if $p \in I_{1}$, is a cyclic $\mathcal{N}_{0}$-module (with generator $t_{\bar{p}}$ ) if $p \in I_{2,3}$, and is not cyclic (with two generators $t_{p}, t_{\bar{p}}$ ) if $p \in I_{4}$. Applying $\theta$ to the above sets and by (3.121), we obtain the claim.

Using Claim 3 and (3.54), by replacing $\mathcal{H}$ by $\theta_{\nu}(\mathcal{H})($ cf. $(3.54))$, we can now suppose $\nu=1$.

Claim 4. There exists $A=\operatorname{diag}\left(A_{1}, \ldots, A_{\iota_{4}}\right) \in G L_{2 \iota_{4}}$, where

$$
A_{p}=\left(\begin{array}{cc}
a_{p}+b_{p} & a_{p} \\
1-a_{p}-b_{p} & 1-a_{p}
\end{array}\right), \quad A_{q}=\left(\begin{array}{cc}
1 & 0 \\
a_{q} & b_{q}
\end{array}\right) \in G L_{2}
$$

for $p \in I_{1} \cup I_{3,4}, q \in I_{2}$, such that $\alpha_{\{p, \bar{p}\}}^{*}=\alpha_{\{p, \bar{p}\}} A_{p}$ for $\alpha \in \Gamma \backslash \Gamma_{1,4}, p \in I_{1,4}$.

Using that $\tau$ is a group isomorphism and applying $\theta$ to

$$
\left[x^{-\sigma_{p}}, x^{\alpha}\right]=\left(\alpha_{\bar{p}}+\eta_{\bar{p}} \alpha_{p}\right) x^{\alpha}
$$

(cf. (3.3) and (3.91)), by (3.80) and (3.81), we obtain

$$
d_{p}\left(\alpha_{\bar{p}}^{*}+\eta_{\bar{p}} \alpha_{p}^{*}\right)=\alpha_{\bar{p}}+\eta_{\bar{p}} \alpha_{p} \text { if } \alpha_{\bar{p}}+\eta_{\bar{p}} \alpha_{p} \neq 0, \alpha \in \Gamma \backslash \Gamma_{1,4}, p \in I_{1,4}
$$


Comparing the coefficients in (3.108), we obtain

$$
\left(\alpha_{p} \gamma_{\bar{p}}-\alpha_{\bar{p}} \gamma_{p}\right) c_{\sigma_{p}+\alpha+\gamma}=c_{\alpha} c_{\gamma}\left(\alpha_{p}^{*} \gamma_{\bar{p}}^{*}-\alpha_{\bar{p}}^{*} \gamma_{p}^{*}\right) \text { if } \alpha_{p} \gamma_{\bar{p}}-\alpha_{\bar{p}} \gamma_{p} \neq 0, \alpha, \gamma, \alpha+\gamma \in \Gamma \backslash \Gamma_{1,4}
$$

Suppose $\alpha \pm \gamma \notin \Gamma_{1,4}$. Replacing $\gamma$ by $-\gamma$ in (3.128), and dividing the result from (3.128), we obtain

$$
c_{-\gamma} c_{\gamma}^{-1}=c_{\sigma_{p}+\alpha-\gamma} c_{\sigma_{p}+\alpha+\gamma}^{-1}=c_{\alpha-\gamma} c_{\alpha+\gamma}^{-1} .
$$

In particular, by taking $\gamma=\sigma_{p}+\lambda_{p}$ (recall (3.97)) and replacing $\alpha$ by $\alpha+\sigma_{p}+\lambda_{p}$, we obtain that

$$
c_{-\sigma_{p}-\lambda_{p}} c_{\alpha+2 \sigma_{p}+2 \lambda_{p}}=c_{\alpha} c_{\sigma_{p}+\lambda_{p}}
$$

holds under some conditions on $\alpha$ (these conditions are linear inequalities on $\alpha_{p}, \alpha_{\bar{p}}$ ). Setting $\gamma=\sigma_{p}+2 \lambda_{p}$ in (3.128) and using (3.130), we obtain

$$
\left(\alpha_{p}\left(-\eta_{\bar{p}}+2 e_{p}\right)-2 \alpha_{\bar{p}}\right) c_{-\sigma_{p}-\lambda_{p}}^{-1}=c_{\sigma_{p}+\lambda_{p}}^{-1} c_{\sigma_{p}+2 \lambda_{p}}\left(\alpha_{p}^{*}\left(-\eta_{\bar{p}}+2 \lambda_{p, \bar{p}}^{*}\right)-\alpha_{\bar{p}}^{*}\left(1+2 \lambda_{p, p}^{*}\right)\right),
$$

holds under some conditions on $\alpha$. Recall from (3.91) that $\eta_{\bar{p}}=0$ if $p \in I_{2}$ and $\eta_{\bar{p}}=-1$ otherwise. Noting that when $p$ is fixed, all coefficients ( $\operatorname{such}$ as $\lambda_{p, p}^{*}$ ) of $\alpha_{p}, \alpha_{\bar{p}}, \alpha_{p}^{*}, \alpha_{\bar{p}}^{*}$ appearing in (3.127) and (3.131) are constant. From (3.127) and (3.131), using (3.99), we can solve $\alpha_{p}^{*}, \alpha_{\bar{p}}^{*}$ as linear combinations of $\alpha_{p}, \alpha_{\bar{p}}$ with the coefficient matrices as required in the claim (i.e., as shown in (3.125)); furthermore, we have $b_{p}=d_{p}^{-1}$. Since $\tau$ is a group isomorphism, the condition on $\alpha$ can be removed, i.e., the claim holds for all $\alpha \in \Gamma$.

Claim 5. In (3.125), $a_{p}=0$ if $p \in I_{3}$.

Let $p \in I_{3}$. We write $\theta\left(t_{p}\right)=b t_{p}^{\prime}+\sum_{\left(0, \varepsilon_{p}\right) \neq(\beta, \underline{j}) \in \Gamma^{\prime} \times \mathcal{J}^{\prime}} b_{\beta, \underline{j}} x^{\beta, \underline{j}}$ for some $b, b_{\beta, \underline{j}} \in \mathbb{F}$. Then we have

$$
\alpha_{\bar{p}} c_{\alpha+\sigma_{p}} x^{\prime \alpha^{*}+\sigma_{p}}=\theta\left(\left[t_{p}, x^{\alpha}\right]\right)=b c_{\alpha} \alpha_{\bar{p}}^{*} x^{\prime \alpha^{*}+\sigma_{p}}+\ldots
$$

for $\alpha \in \Gamma \backslash \Gamma_{1,4}$, where the missed terms do not contain $x^{\prime \alpha^{*}+\sigma_{p}}$. Thus by (3.125), we have

$$
\alpha_{\bar{p}} c_{\alpha+\sigma_{p}}=b c_{\alpha}\left(a_{p} \alpha_{p}+\left(1-a_{p}\right) \alpha_{\bar{p}}\right)
$$

Hence $b \neq 0$. Take $0 \neq \alpha \in \mathbb{F} \varepsilon_{p} \cap \Gamma$ (then $\left.\alpha \notin \Gamma_{1,4}\right)$, we obtain $a_{p}=0$. This also proves (3.43) and (3.44).

Claim 6. Denote $\sigma=\sum_{p \in I_{1,4}} \sigma_{p}$. For any $\alpha \in \Gamma$ with $\alpha_{J_{1,4}} \neq \sigma$, we have $\theta\left(x^{\alpha}\right)=c_{\alpha} x^{\prime \alpha^{*}}$ for some $c_{\alpha} \in \mathbb{F}^{\times}$.

Assume that $\alpha \in \Gamma_{1,4}$ with $\alpha_{J_{1,4}} \neq \sigma$. Then by (2.26), we can always choose $\beta=$ $\beta_{p} \varepsilon_{p}+\beta_{\bar{p}} \varepsilon_{\bar{p}} \in \Gamma \backslash \Gamma_{1,4}$ for some $p \in I_{1,4}$, such that

$$
a=\beta_{p}\left(\alpha_{\bar{p}}-\beta_{\bar{p}}+\eta_{p}\right)-\beta_{\bar{p}}\left(\alpha_{p}-\beta_{p}-1\right) \neq 0 .
$$

Then $\beta, \alpha-\beta-\sigma_{p} \notin \Gamma_{1,4}$ and $\beta^{*} \in \widetilde{\Gamma}_{p}^{\prime}$ (where $\widetilde{\Gamma}_{p}^{\prime}$ is a similar notation as in (3.107)). We have

$$
\theta\left(x^{\alpha}\right)=a^{-1} \theta\left(\left[x^{\beta}, x^{\alpha-\beta-\sigma_{p}}\right]\right)=a^{-1} c_{\beta} c_{\alpha-\beta-\sigma_{p}}\left[x^{\beta^{*}}, x^{\prime \alpha^{*}-\beta^{*}-\sigma_{p}}\right] \in \mathbb{F} x^{\prime \alpha^{*}} .
$$


By (3.7) and Lemma 3.1, we have

$$
\theta\left(t_{p}\right) \in \mathcal{H}^{\prime F} \subset \operatorname{span}\left(H_{1}^{\prime} \cup H_{3}^{\prime}\right)=\sum_{q \in I_{1,4}^{\prime}} \mathbb{F} x^{\prime-\sigma_{q}}+\sum_{r \in \bar{I}_{5,6}^{\prime}} \mathbb{F} t_{r}^{\prime}+H_{3}^{\prime},
$$

for $p \in \bar{I}_{5,6}$. Thus, using notations (2.22) and (3.76), we have (also recall notations (3.60) and $(3.61))$

$$
\theta^{-1}\left(\bar{t}_{\bar{I}_{5,6}^{\prime}}^{\prime}\right) \equiv\left(x^{-\sigma}\right)_{I_{1,4}} F_{1}+\bar{t}_{\bar{I}_{5,6}} F_{2}\left(\bmod H_{3}\right)
$$

for some

$$
\begin{gathered}
F_{1}=\left(a_{p, q}\right)_{p \in I_{1,4}, q \in \bar{I}_{5,6}^{\prime}} \in M_{\iota_{4} \times\left(\ell_{5}^{\prime}+\ell_{6}^{\prime}\right)}, \\
F_{2}=\left(b_{p, q}\right)_{p \in \bar{I}_{5,6}, q \in \bar{I}_{5,6}^{\prime}} \in G L_{\ell_{5}+\ell_{6}},
\end{gathered}
$$

(in particular $\ell_{5}+\ell_{6}=\ell_{5}^{\prime}+\ell_{6}^{\prime}$ ).

Claim 7. We have

$$
\begin{gathered}
a_{p, q}=0 \text { if } p \in I_{3,4}, q \in \bar{I}_{5}^{\prime}, \\
b_{p, q}=0 \text { if } p \notin I_{5}, q \in \bar{I}_{5}^{\prime},
\end{gathered}
$$

which implies $\left(\ell_{5}, \ell_{6}\right)=\left(\ell_{5}^{\prime}, \ell_{6}^{\prime}\right)$ and $I_{i}=I_{i}^{\prime}$ for $i=5,6$.

Note that the center of $\mathcal{M}$ is $\mathcal{C}=\left\{x^{\alpha} \in \Gamma \mid \alpha=\alpha_{I_{5,6}}\right\}$. Denote the centralizer $C_{\mathcal{H}}(\mathcal{C})=$ $\{u \in \mathcal{H} \mid[u, \mathcal{C}]=0\}$. It is straightforward to check that

$$
\left\{t_{p} \mid p \in I_{2,3} \cup J_{4} \cup I_{6}\right\} \subset C_{\mathcal{H}}(\mathcal{C}) \subset \operatorname{span}\left\{x^{\alpha, \underline{i}} \mid \underline{i}_{\bar{I}_{5,6}}=0\right\} .
$$

For $p \in I_{1,4},(3.142)$ implies that $\left.\operatorname{ad}_{x^{-\sigma_{p}}}\right|_{C_{\mathcal{H}}(\mathcal{C})}$ is semi-simple if and only if $p \in I_{1,2}$, and $\left.\operatorname{ad}_{t_{q}}\right|_{C_{\mathcal{H}}(C)}$ is semi-simple for $q \in \bar{I}_{5}$ by (3.142) and is not semi-simple for $q \in \bar{I}_{6}$. Moreover, by (3.1), for $p \in \bar{I}_{5,6}, \operatorname{ad}_{t_{p}}$ is semi-simple if and only if $p \in \bar{I}_{5}$. We obtain the claim.

By (3.140) and (3.141), we can write $F_{1}$ and $F_{2}$ is the forms

$$
F_{1}=\left(\begin{array}{cc}
B_{1,5} & B_{1,6} \\
B_{2,5} & B_{2,6} \\
0 & B_{3,6}
\end{array}\right), \quad F_{2}=\left(\begin{array}{cc}
B_{5,5} & B_{5,6} \\
0 & B_{6,6}
\end{array}\right)
$$

such that all $B_{i, j}$ have the forms in (3.46) and (3.48).

For any $\alpha \in \Gamma$, we denote

$$
\widehat{\alpha}=\left(\alpha_{\overline{1}}+\eta_{\overline{1}} \alpha_{1}, \ldots, \alpha_{\bar{\iota}_{4}}+\eta_{\bar{\iota}_{4}} \alpha_{\iota_{4}}\right) \in \mathbb{F}^{\iota_{4}},
$$

(cf. (3.91)). For $\alpha \in \Gamma \backslash \Gamma_{1,4}$, applying $\theta^{-1}$ to

$$
\alpha_{I_{5,6}}^{*} c_{\alpha}^{-1} x^{\prime \alpha^{*}}=\left[\bar{t}_{I_{5,6}}^{\prime}, c_{\alpha}^{-1} x^{\prime \alpha^{*}}\right],
$$

(cf. (3.4)), using (3.137), and noting that $\left[H_{3}, \mathcal{M}\right]=0$, we obtain

$$
\alpha_{I_{5,6}}^{*} x^{\alpha}=\left[\left(x^{-\sigma}\right)_{I_{1,4}} F_{1}+\bar{t}_{\bar{I}_{5,6}} F_{2}, x^{\alpha}\right]=\left(\widehat{\alpha} F_{1}+\alpha_{I_{5,6}} F_{2}\right) x^{\alpha},
$$


that is

$$
\alpha_{I_{5,6}}^{*}=\widehat{\alpha} F_{1}+\alpha_{I_{5,6}} F_{2}
$$

holds for all $\alpha \in \Gamma \backslash \Gamma_{1,4}$ and so holds for all $\alpha \in \Gamma$ since $\tau: \alpha \mapsto \alpha^{*}$ is an isomorphism. From this and (3.143), we obtain formulas (3.43)-(3.48) (cf. (3.49)).

Claim 8. $\ell_{7}=\ell_{7}^{\prime}$.

Observe from (3.9) that

$$
\begin{gathered}
H_{3}=C_{\mathcal{H}}(\mathcal{M}) \quad(\text { the centralizer of } \mathcal{M}), \\
\operatorname{span}\left\{x^{\alpha, \underline{i}} \in H_{3} \mid \underline{i}_{J_{7}}=0\right\}=C\left(H_{3}\right) \quad\left(\text { the center of } H_{3}\right) .
\end{gathered}
$$

By exchanging $\mathcal{H}$ with $\mathcal{H}^{\prime}$ if necessary, we can suppose $\ell_{7} \leq \ell_{7}^{\prime}$. As in the proof of sufficiency, we can construct an embedding $\bar{\theta}: \mathcal{H} \rightarrow \mathcal{H}^{\prime}$ such that

$$
\bar{\theta}\left(x^{\alpha}\right)=\theta\left(x^{\alpha}\right), \quad \bar{\theta}\left(\bar{t}_{\bar{I}_{5,6}}\right) \equiv \theta\left(\bar{t}_{\bar{I}_{5,6}}\right)\left(\bmod H_{3}^{\prime}\right)
$$

(cf. Claim 6 and (3.137), note that using (3.137), we can now obtain that Claim 6 holds for all $\alpha \in \Gamma$ if $\ell_{5}+\ell_{6} \neq 0$ ). Thus by identifying $\mathcal{H}$ with $\bar{\theta}(\mathcal{H})$, we can assume that $\mathcal{H}$ is a subalgebra of $\mathcal{H}^{\prime}$ such that there exists an isomorphism $\theta$ satisfying

$$
\theta\left(x^{\alpha}\right)=x^{\alpha}, \quad \theta\left(t_{\bar{p}}\right) \equiv t_{\bar{p}}\left(\bmod H_{3}^{\prime}\right) \quad \text { for } \quad \alpha \in \Gamma, p \in I_{5,6} \text {. }
$$

By restricting $\theta$ to $H_{3}$, we want to prove

$$
\begin{gathered}
\theta\left(t_{p}\right)=t_{p}+c_{p} \text { for } p \in I_{6} \text { and some } c_{p} \in \mathbb{F} \\
\theta\left(x^{\alpha, \underline{i}} \underline{t}^{j}\right)=x^{\alpha} \prod_{p \in I_{6}}\left(\theta\left(t_{p}\right)\right)^{i_{p}} \prod_{q \in J_{7}}\left(\theta\left(t_{q}\right)\right)^{j_{q}} \quad \text { for } \quad \alpha=\alpha_{I_{5,6}}, \underline{i}=\underline{i}_{I_{6}}, \underline{j}=\underline{j}_{J_{7}} .
\end{gathered}
$$

To prove (3.152), first by (3.149), we have $c_{p}=\theta\left(t_{p}\right)-t_{p} \in C\left(H_{3}^{\prime}\right)$. Then by (3.151), we have

$$
\left[t_{\bar{q}}, c_{p}\right]=\theta\left(\left[\theta^{-1}\left(t_{\bar{q}}\right), t_{p}\right]\right)-\left[t_{\bar{q}}, t_{p}\right]=0
$$

where the second equality follows from the fact that $\theta^{-1}\left(t_{\bar{q}}\right)=t_{\bar{q}}\left(\bmod H_{3}\right)$ and $\left[H_{3}, t_{p}\right]=0$. From (3.154), we obtain that $c_{p} \in \mathbb{F}$. Thus we have (3.152). Similarly, we have

$$
\theta\left(x^{\alpha, \varepsilon_{p}}\right)=x^{\alpha}\left(t_{p}+c_{\alpha, p}\right) \text { for } p \in I_{6} \text { and some } c_{\alpha, p} \in \mathbb{F} .
$$

By considering $\theta\left(\left[x^{\alpha}, t_{p} t_{\bar{p}}\right]\right)=\left[\theta\left(x^{\alpha}\right), \theta\left(t_{p} t_{\bar{p}}\right)\right]$, we see that $c_{\alpha, p}=c_{p}$, and we obtain

$$
\theta\left(t_{p} t_{\bar{p}}\right)=\left(t_{p}+c_{p}\right) t_{\bar{p}}+u_{p} \text { for } \quad p \in I_{6} \text { and some } u_{p} \in C_{\mathcal{H}^{\prime}}\left(C\left(H_{3}^{\prime}\right)\right) .
$$

From this and (3.152), we can deduce

$$
\theta\left(x^{\alpha, \varepsilon_{p}}\right)=x^{\alpha}\left(t_{p}+c_{p}\right) \text { for } p \in I_{6}
$$

Similar to (3.156), we have

$$
\theta\left(x^{-\sigma_{p}, \varepsilon_{p}+\varepsilon_{\bar{p}}}\right)=x^{-\sigma_{p}, \varepsilon_{\bar{p}}}\left(t_{p}+c_{p}\right)+u_{p}^{\prime} \quad \text { for } \quad p \in I_{6} \text { and some } u_{p}^{\prime} \in C_{\mathcal{H}^{\prime}}\left(C\left(H_{3}^{\prime}\right)\right) .
$$


Now from (3.152), (3.155)-(3.158), we can obtain (3.153) by induction on $|\underline{i}|$ in case $\underline{j}=0$.

Assume that (3.153) holds for all $\underline{j}$ with $|\underline{j}|<n$, where $n \geq 1$. We denote by $A_{\alpha, \underline{i}, \underline{j}}$ the difference between the left-hand side and the right-hand side of (3.153). Then the inductive assumption says that $A_{\alpha, \underline{i}, \underline{j}}=0$ if $|\underline{j}|<n$. Now suppose $|\underline{j}|=n$. Say $j_{r} \geq 1$ for some $r \in I_{7}$ (the proof is similar if $r \in \bar{I}_{7}$ ). Let $\underline{k}=\underline{j}-\varepsilon_{r}+\varepsilon_{\bar{r}}$. Then we have

$$
\begin{aligned}
{\left[\theta\left(t_{r}\right), A_{\alpha, \underline{i}, \underline{k}]}\right]=} & \theta\left(\left[t_{r}, x^{\alpha, \underline{i}} t^{\underline{k}}\right]\right)-\theta\left(\left[t_{r}, \theta^{-1}\left(x^{\alpha}\right)\right]\right) \prod_{p \in I_{6}}\left(\theta\left(t_{p}\right)\right)^{i_{p}} \prod_{q \in J_{7}}\left(\theta\left(t_{q}\right)\right)^{k_{q}} \\
& -x^{\alpha}\left[\theta\left(t_{r}\right), \prod_{p \in I_{6}}\left(\theta\left(t_{p}\right)\right)^{i_{p}} \prod_{q \in J_{7}}\left(\theta\left(t_{q}\right)\right)^{k_{q}}\right] \\
= & \left(j_{\bar{r}}+1\right)\left(\theta\left(x^{\alpha, \underline{i}-\underline{j}-\varepsilon_{r}}\right)-x^{\alpha} \prod_{p \in I_{6}}\left(\theta\left(t_{p}\right)\right)^{i_{p}} \prod_{q \in J_{7}}\left(\theta\left(t_{q}\right)\right)^{j_{q}-\delta_{q, r}}\right) \\
= & \left(j_{\bar{r}}+1\right) A_{\alpha, \underline{i}, \underline{j}-\varepsilon_{r}}=0,
\end{aligned}
$$

where the first equality follows from (1.1), the second equality follows from (1.1) and (3.151). By (1.1) and (3.159), we obtain

$$
\left[\theta\left(t_{r}^{2}\right), A_{\alpha, \underline{i}, \underline{k}}\right]=\theta\left(\left[t_{r}^{2}, \theta^{-1}\left(A_{\alpha, \underline{i}, \underline{k}}\right)\right]\right)=2 \theta\left(t_{r}\left[t_{r}, \theta^{-1}\left(A_{\alpha, \underline{i}, \underline{k}}\right)\right]\right)=0 .
$$

On the other hand, exactly similar to (3.159), we have

$$
\left[\theta\left(t_{r}^{2}\right), A_{\alpha, \underline{i}, \underline{k}}\right]=2\left(j_{\bar{r}}+1\right) A_{\alpha, \underline{i}, \underline{j}} .
$$

Now (3.160) and (3.161) show that $A_{\alpha, \underline{i}, j}=0$. This proves (3.153). By (3.152), (3.153) and by identifying $C\left(H_{3}\right)$ with $C\left(H_{3}^{\prime}\right)$ using the isomorphism, we see that $\theta$ is an associative algebra isomorphism $H_{3} \rightarrow H_{3}^{\prime}$ over the domain ring $C\left(H_{3}\right)$. From this we obtain $\ell_{7}=\ell_{7}^{\prime}$ since $2 \ell_{7}$ is the transcendental degree of $H_{3}$ over the domain ring $C\left(H_{3}\right)$. This completes the proof of Theorem 3.6.

\section{Derivations}

In this section, we shall determine the structure of the derivation algebra of the Hamiltonian Lie algebra $\mathcal{H}=\mathcal{H}(\underline{\ell}, \Gamma)$. As pointed in $[\mathrm{F}]$, the significance of derivations for Lie theory primarily resides in their affinity to low dimensional cohomology groups, their determination therefore frequently affords insight into structural features of Lie algebras which do not figure prominently in the defining properties. Some general results concerning derivations of graded Lie algebras were established in $[\mathrm{F}]$. However in our case the algebras are in general nongraded, the results in $[\mathrm{F}]$ can not be applied to our case here. Thus we try a different method to determine derivations of the Hamiltonian Lie algebras $\mathcal{H}$. Our method is also different from that used in [OZ].

Recall that a derivation $d$ of the Lie algebra $\mathcal{H}$ is a linear transformation on $\mathcal{H}$ such that

$$
d\left(\left[u_{1}, u_{2}\right]\right)=\left[d\left(u_{1}\right), u_{2}\right]+\left[u_{1}, d\left(u_{2}\right)\right] \text { for } u_{1}, u_{2} \in \mathcal{H} .
$$

Denote by Der $\mathcal{H}$ the space of the derivations of $\mathcal{H}$, which is a Lie algebra. Moreover, $\operatorname{ad}_{\mathcal{H}}$ is an ideal. Elements in $\operatorname{ad}_{\mathcal{H}}$ are called inner derivations, while elements in Der $\mathcal{H} \backslash \operatorname{ad}_{\mathcal{H}}$ are called outer derivations. 
We can embed $\mathcal{H}$ into a larger Lie algebra $\widetilde{\mathcal{H}}$ such that $\widetilde{\mathcal{H}}$ has a basis $\left\{x^{\alpha, \underline{i}} \mid(\alpha, \underline{i}) \in \Gamma \times \mathbb{N}^{2 \iota_{7}}\right\}$ (i.e., in $\widetilde{\mathcal{H}}$, we replace $\mathcal{J}$ by $\mathbb{N}^{2 \iota_{7}}$, cf. (2.27), and we have (3.1) with the last three summands running over $p \in I_{1,6}, p \in I_{1,4}$ and $p \in I$ respectively). Then for $p \in J_{1} \cup \bar{I}_{2,3} \cup I_{5}$, clearly, $t_{p} \notin \mathcal{H}$, but $\left[t_{p}, \mathcal{H}\right] \subset \mathcal{H}$. Thus

$$
d_{p}=\left.\operatorname{ad}_{t_{p}}\right|_{\mathcal{H}} \text { for } p \in J_{1} \cup \bar{I}_{2,3} \cup I_{5},
$$

defines an outer derivation of $\mathcal{H}$. For $p \in I_{2,3} \cup J_{4} \cup \bar{I}_{5} \cup J_{6,7}$, obviously, $\partial_{t_{p}}$ is a derivation of $\mathcal{H}$ (cf. (2.27), (2.35) and (3.1)). For $p \in J$, we define $\operatorname{sgn}(p)=1$ if $p \in I$ and $\operatorname{sgn}(p)=-1$ if $p \in \bar{I}$. Then

$$
\partial_{t_{p}}=\operatorname{sgn}(p) \operatorname{ad}_{t_{\bar{p}}} \text { for } \quad p \in \bar{I}_{5} \cup J_{6,7} .
$$

Define $d_{0}\left(x^{\alpha, \underline{i}}\right)=\left(\sum_{p \in I_{1,4}} \alpha_{p}+1\right) x^{\alpha, \underline{i}}$ for $(\alpha, \underline{i}) \in \Gamma \times \mathcal{J}$. It is straightforward to verify that $d_{0}$ is an outer derivation of $\mathcal{H}$. Denote $\sigma=\sum_{p \in I_{1,4}} \sigma_{p}$. If $\iota_{7}=\ell_{1}$, then $\mathcal{H}=[\mathcal{H}, \mathcal{H}]+\mathbb{F} x^{\sigma}$, and we can define an outer derivation $d_{0}^{\prime}$ by setting

$$
d_{0}^{\prime}([\mathcal{H}, \mathcal{H}])=0, \quad d_{0}^{\prime}\left(x^{\sigma}\right)=1_{\mathcal{H}} .
$$

If $\iota_{7} \neq \ell_{1}$, we set $d_{0}^{\prime}=0$.

We denote by $\operatorname{Hom}_{\mathbb{Z}}^{+}(\Gamma, \mathbb{F})$ the set of group homomorphisms $\mu: \Gamma \rightarrow \mathbb{F}$ such that $\mu\left(\sigma_{p}\right)=0$ for $p \in I_{1,4}$. For $\mu \in \operatorname{Hom}_{\mathbb{Z}}^{+}(\Gamma, \mathbb{F})$, we define a linear transformation $d_{\mu}$ on $\mathcal{H}$ by

$$
d_{\mu}\left(x^{\alpha, \underline{i}}\right)=\mu(\alpha) x^{\alpha, \underline{i}} \text { for }(\alpha, \underline{i}) \in \Gamma \times \mathcal{J} .
$$

Clearly, by (3.1), $d_{\mu}$ is a derivation of $\mathcal{H}$. We identify $\operatorname{Hom}_{\mathbb{Z}}^{+}(\Gamma, \mathbb{F})$ with a subspace of Der $\mathcal{H}$ by $\mu \mapsto d_{\mu}$. For $p \in I_{1,6}$, we define $\mu_{p} \in \operatorname{Hom}_{\mathbb{Z}}^{+}(\Gamma, \mathbb{F})$ by

$$
\mu_{p}(\alpha)= \begin{cases}\alpha_{\bar{p}}+\eta_{\bar{p}} \alpha_{p} & \text { if } \quad p \in I_{1,4}, \\ \alpha_{p} & \text { if } \quad p \in I_{5,6}\end{cases}
$$

for $\alpha \in \Gamma$ (cf. (3.91)). By (3.3) and (3.4), we have

$$
\begin{aligned}
\operatorname{ad}_{x^{-\sigma_{p}}}=\left\{\begin{array}{lll}
d_{\mu_{p}} & \text { if } & p \in I_{1,2}, \\
d_{\mu_{p}}+\partial_{t_{p}} & \text { if } & p \in I_{3}, \\
d_{\mu_{p}}+\partial_{t_{p}}-\partial_{t_{\bar{p}}} & \text { if } & p \in I_{4},
\end{array}\right. \\
\operatorname{ad}_{t_{\bar{q}}}=\left\{\begin{array}{lll}
-d_{\mu_{q}} & \text { if } & q \in I_{5}, \\
-d_{\mu_{q}}-\partial_{t_{q}} & \text { if } & q \in I_{6} .
\end{array}\right.
\end{aligned}
$$

We fix a subspace $\operatorname{Hom}_{\mathbb{Z}}^{*}(\Gamma, \mathbb{F})$ of $\operatorname{Hom}_{\mathbb{Z}}^{+}(\Gamma, \mathbb{F})$ such that

$$
\operatorname{Hom}_{\mathbb{Z}}^{+}(\Gamma, \mathbb{F})=\operatorname{Hom}_{\mathbb{Z}}^{*}(\Gamma, \mathbb{F}) \oplus \operatorname{span}\left\{\mu_{p} \mid p \in I_{1,6}\right\}
$$

is a direct sum as vector spaces. Since $\operatorname{ad}_{\mathbb{F}}=0$, we set $\mathcal{H}^{*}=\operatorname{span}\left\{x^{\alpha, \underline{i}} \mid(0,0) \neq(\alpha, \underline{i}) \in \Gamma \times \mathcal{J}\right\}$.

Theorem 4.1. The derivation algebra Der $\mathcal{H}$ is spanned by

$$
d_{0}^{\prime}, d_{p}, \partial_{t_{q}}, d_{\mu}, a d_{\mathcal{H}^{*}} \quad \text { for } \quad p \in\{0\} \cup J_{1} \cup \bar{I}_{2,3} \cup I_{5}, q \in I_{2,3} \cup J_{4}, \mu \in \operatorname{Hom}_{\mathbb{Z}}^{*}(\Gamma, \mathbb{F}) \text {. }
$$


Furthermore, we have the following vector space decomposition as a direct sum of subspaces:

$$
\operatorname{Der} \mathcal{H}=\left(\left(\mathbb{F} d_{0}^{\prime}+\sum_{p \in\{0\} \cup J_{1} \cup \bar{I}_{2,3} \cup I_{5}} \mathbb{F} d_{p}\right) \oplus \sum_{q \in I_{2,3} \cup J_{4}} \mathbb{F} \partial_{t_{q}} \oplus \operatorname{Hom}_{\mathbb{Z}}^{*}(\Gamma, \mathbb{F})\right) \oplus a d_{\mathcal{H}^{*}} .
$$

In particular, all derivations of the classical Hamiltonian Lie algebras $\mathcal{H}(\ell)(c f .(1.3))$ are inner.

Proof. First note that in [OZ], $d_{0}$ was written as a derivation of the form $d_{\mu}$ with $\mu$ satisfying $\mu\left(\sigma_{p}\right)=\mu\left(\sigma_{1}\right)$ for $p \in I_{1,4}$. Let $d \in \operatorname{Der} \mathcal{H}$ and let $D$ be the subspace of Der $\mathcal{H}$ spanned by the elements in (4.10). Note that $D \supset \operatorname{Hom}_{\mathbb{Z}}^{+}(\Gamma, \mathbb{F})$ by (4.7) and (4.8). We shall prove that after a number of steps in each of which $d$ is replaced by $d-d^{\prime}$ for some $d^{\prime} \in D$ the 0 derivation is obtained and thus proving that $d \in D$. This will be done by a number of claims.

Claim 1. We can suppose (i) $d(1)=0$, (ii) $d\left(x^{-\sigma_{p}}\right)=0$ for $p \in I_{3,4}$, (iii) $d\left(x^{\varepsilon_{q}}\right)=d\left(t_{r}\right)=0$ for $q \in I_{5,6}, r \in \bar{I}_{6} \cup J_{7}$.

By replacing $d$ by $d-d(1) d_{0}$, we can suppose $d(1)=0$. For any $(\alpha, \underline{i}) \in \Gamma \times \mathcal{J}$, we write

$$
\begin{gathered}
d\left(x^{\alpha, \underline{i}}\right)=\sum_{(\beta, \underline{j}) \in M_{\alpha, \underline{i}}} c_{\alpha, \underline{\underline{i}}}^{(\beta, \underline{j})} x^{\alpha+\beta, \underline{j}} \text { for some } c_{\alpha, \underline{\underline{i}}}^{(\beta, \underline{j})} \in \mathbb{F} \text {, where } \\
M_{\alpha, \underline{\underline{i}}}=\left\{(\beta, \underline{j}) \in \Gamma \times \mathcal{J} \mid c_{\alpha, \underline{\underline{i}}}^{(\beta, \underline{j})} \neq 0\right\},
\end{gathered}
$$

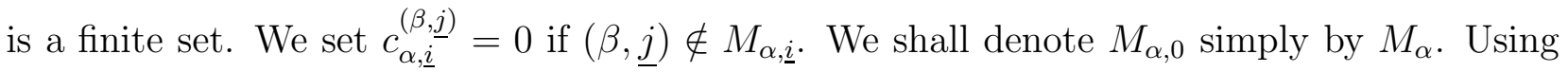
inductive assumption, suppose we have proved that $d\left(x^{-\sigma_{r}}\right)=0$ for $r \in I_{3,4}$ and $r<p$. Let $(\beta, \underline{j}) \in M_{-\sigma_{p}}$. Using (3.3), one can deduce by induction on $|\underline{j}|$ that

$$
x^{-\sigma_{p}+\beta, \underline{j}}=\left[u_{\beta, \underline{j}}, x^{-\sigma_{p}}\right] \text { for some } u_{\beta, \underline{j}} \in \mathcal{H},
$$

such that $u_{\beta, \underline{j}}$ has the following form

$$
u_{\beta, \underline{j}}=\sum_{k, \ell \in \mathbb{Z}} b_{k, \ell} x^{-\sigma_{p}+\beta, \underline{j}+k \varepsilon_{p}+\ell \varepsilon_{\bar{p}}} \text { for some } \quad b_{k, \ell} \in \mathbb{F},
$$

(recall convention (2.34)). Thus we can take

$$
u=\sum_{(\beta, \underline{j}) \in M_{-\sigma_{p}}} c_{-\sigma_{p}, 0}^{(\beta, j)} u_{\beta, \underline{j}} \in \mathcal{H} \text { such that }\left(d-\operatorname{ad}_{u}\right)\left(x^{-\sigma_{p}}\right)=0,
$$

Applying $d$ to $\left[x^{-\sigma_{r}}, x^{-\sigma_{p}}\right]=0$, we obtain

$$
\sum_{(\beta, \underline{j}) \in M_{-\sigma_{p}}} c_{-\sigma_{p}, 0}^{(\beta, j)}\left[x^{-\sigma_{r}}, x^{-\sigma_{p}+\beta, \underline{j}}\right]=0 \quad \text { for } \quad r \in I_{3,4}, r<p
$$

i.e.,

$$
c_{-\sigma_{p}, 0}^{(\beta, j)}\left(\beta_{r}-\beta_{\bar{r}}\right)-c_{-\sigma_{p}, 0}^{\left(\beta, j+\varepsilon_{\bar{T}}\right)}\left(j_{\bar{r}}+1\right)+c_{-\sigma_{p}, 0}^{\left(\beta, j+\varepsilon_{r}\right)}\left(j_{r}+1\right)=0,
$$

for $r \in I_{3,4}, r<p$, from this and by induction on $j_{r}+j_{\bar{r}}$ ranging from $\max \left\{k_{r}+k_{\bar{r}} \mid(\beta, \underline{k}) \in\right.$ $\left.M_{-\sigma_{p}}\right\}$ down to zero, we obtain

$$
\beta_{r}=\beta_{\bar{r}}, j_{r}=j_{\bar{r}}=0 \quad \text { for } \quad(\beta, \underline{j}) \in M_{-\sigma_{p}}, r \in I_{3,4}, r<p .
$$


Then (4.15), (4.16) and (4.19) show that $\operatorname{ad}_{u}\left(x^{-\sigma_{r}}\right)=0$ for $r \in I_{3,4}, r<p$. Thus if we replace $d$ by $d-\operatorname{ad}_{u}$, we have $d\left(x^{-\sigma_{r}}\right)=0$ for $r \in I_{3,4}, r \leq p$. This proves Claim 1(ii). Note that for $v=x^{\varepsilon_{q}}, q \in I_{5,6}$, or $v=t_{r}, r \in \bar{I}_{6} \cup J_{7}$, we have $\operatorname{ad}_{v}(\mathcal{H})=\mathcal{H}$. Thus similar to the above proof, we have Claim 1(iii).

Note that for $(\beta, \underline{j}) \in \Gamma \times \mathcal{J}$, by $(3.3)$ and $(3.4)$, we have

$$
\begin{gathered}
\left(\beta_{\bar{p}}+\eta_{\bar{p}} \beta_{p}\right) x^{-\sigma_{p}+\beta, \underline{j}}=\left[x^{-\sigma_{p}+\beta, \underline{j}}, x^{-\sigma_{p}}\right] \text { for } p \in I_{1,2}, \\
\left(-1+\beta_{p}\right) e_{p} x^{\lambda_{p}+\beta, \underline{j}}=\left[x^{-\sigma_{p}+\beta, \underline{j}}, x^{\lambda_{p}}\right]+j_{p} x^{\lambda_{p}+\beta, \underline{j}-\varepsilon_{p}} \quad \text { for } \quad p \in I_{1,4},
\end{gathered}
$$

(recall notations $\lambda_{p}, p \in I_{1,4}$ in $(3.97)$ ), and

$$
\beta_{p} x^{\beta, \underline{j}}=\left[x^{\beta, \underline{j}}, t_{\bar{p}}\right] \text { for } p \in I_{5}
$$

Claim 2. By replacing $d$ by $d-d^{\prime}$ for some $d^{\prime} \in D$, we can suppose

$$
\begin{gathered}
\beta_{\bar{p}}+\eta_{\bar{p}} \beta_{p}=0 \quad \text { for } \quad(\beta, \underline{j}) \in M_{-\sigma_{p}}, p \in I_{1,2} \\
\beta_{p}=1 \text { for }(\beta, \underline{j}) \in M_{\lambda_{p}}, p \in I_{1,4} \\
\beta_{p}=0 \quad \text { for } \quad(\beta, \underline{j}) \in M_{0, \varepsilon_{\bar{p}}}, p \in I_{5} .
\end{gathered}
$$

The proof of (4.23) is similar to that of Claim 1. To prove (4.24), suppose we have proved

$$
\beta_{r}=1 \text { for }(\beta, \underline{j}) \in M_{\lambda_{r}}, i \in I_{1,4}, r<p .
$$

To see how the proof works, for simplicity, we assume that $p \in I_{1}$ (the proof for $p \in I_{2,4}$ is exactly similar). Then the second term on the right-hand side of (4.21) vanishes. Let

$$
u=\sum_{(\beta, \underline{j}) \in M_{\lambda_{p}}, \beta_{p} \neq 1} c_{\lambda_{p}, 0}^{(\beta, j)}\left(\left(-1+\beta_{p}\right) e_{p}\right)^{-1} x^{-\sigma_{p}+\beta} .
$$

Then by replacing $d$ by $d-\operatorname{ad}_{u}$, from (4.21), we see that (4.24) holds for $p$. We want to prove that after this replacement, Claim 1, (4.23) and (4.26) still hold. It suffices to prove

$$
\left[u, x^{-\sigma_{q}}\right]=\left[u, x^{\varepsilon_{q^{\prime}}}\right]=\left[u, t_{q^{\prime \prime}}\right]=\left[u, x^{\lambda_{r}}\right]=0,
$$

for $q \in I_{1,4}, q^{\prime} \in I_{5,6}, q^{\prime \prime} \in \bar{I}_{6} \cup J_{7}, r \in I_{1,4}, r<p$.

We have

$$
\begin{aligned}
- & e_{p} \sum_{(\beta, \underline{j}) \in M_{\lambda_{p}}} c_{\lambda_{p}, 0}^{(\beta, \underline{j})} x^{\lambda_{p}+\beta, \underline{j}}=-e_{p} d\left(x^{\lambda_{p}}\right)=d\left(\left[x^{-\sigma_{p}}, x^{\lambda_{p}}\right]\right. \\
= & \sum_{(\beta, \underline{j}) \in M_{-\sigma_{p}}} c_{-\sigma_{p}, 0}^{(\beta, \underline{j})}\left(-1+\beta_{p}\right) x^{\lambda_{p}+\beta, \underline{j}}+\sum_{(\beta, \underline{j}) \in M_{\lambda_{p}}} c_{\lambda_{p}, \underline{0}}^{(\beta, j)}\left(\beta_{p}-\beta_{\bar{p}}-e_{p}\right) x^{\lambda_{p}+\beta, \underline{j}} .
\end{aligned}
$$

This gives

$$
\beta_{p}-\beta_{\bar{p}}=\left(c_{\lambda_{p}, 0}^{(\beta, j)}\right)^{-1}\left(\beta_{p}-1\right) c_{-\sigma_{p}, 0}^{(\beta, j)} \quad \text { for } \quad(\beta, \underline{j}) \in M_{\lambda_{p}}
$$


If $(\beta, \underline{j}) \notin M_{-\sigma_{p}}$, then the right-hand side of (4.30) is zero; on the other hand, if $(\beta, \underline{j}) \in M_{-\sigma_{p}}$, then (4.23) gives $\beta_{p}-\beta_{\bar{p}}=0$. In any case, we have $\beta_{p}-\beta_{\bar{p}}=0$ for $(\beta, \underline{j}) \in M_{\lambda_{p}}$. Thus by (4.27),

$$
\left[u, x^{-\sigma_{p}}\right]=\sum_{(\beta, j) \in M_{\lambda_{p}, \beta_{p} \neq 1}} c_{\lambda_{p}, 0}^{(\beta, j)}\left(\left(-1+\beta_{p}\right) e_{p}\right)^{-1}\left(\beta_{p}-\beta_{\bar{p}}\right) x^{-\sigma_{p}+\beta}=0 .
$$

Similarly, we can prove other equations in (4.28). This proves (4.24). Similarly, we have (4.25).

Claim 3. By replacing $d$ by $d-\sum_{p \in J_{1} \cup \bar{I}_{2,3} \cup I_{5}} a_{p} d_{p}-d_{\mu}$ for some $a_{p} \in \mathbb{F}$ and some $\mu \in \operatorname{Hom}_{\mathbb{Z}}^{+}(\Gamma, \mathbb{F})$, we can suppose $d\left(x^{-\sigma_{p}}\right)=d\left(x^{\lambda_{q}}\right)=d\left(t_{r}\right)=0$ for $p \in I_{1,2}, q \in I_{1,4}, r \in I_{5}$.

Again for simplicity, we prove that after some replacement, $d\left(x^{-\sigma_{p}}\right)=d\left(x^{\lambda_{p}}\right)=0$ for $p \in I_{1}$. Defining $\mu \in \operatorname{Hom}_{\mathbb{Z}}^{+}(\Gamma, \mathbb{F})$ by $\mu(\alpha)=c_{\lambda_{p}, 0}^{(0,0)} e_{p}^{-1}\left(\alpha_{\bar{p}}-\alpha_{p}\right)$, and by replacing $d$ by $d-d_{\mu}$, we obtain $c_{\lambda_{p}, 0}^{(0,0)}=0$ (recall (4.12) that $c_{\alpha, \underline{\underline{i}}}^{(\beta, j)}$ is the coefficient of $x^{\alpha+\beta, \underline{j}}$, not that of $x^{\beta, \underline{j}}$ ). Obviously, this replacement does not affect the result we have obtained so far. Recalling the definition of $d_{p}$ in (4.2), we have

$$
d_{p}\left(x^{-\sigma_{p}}\right)=\left[t_{p}, x^{-\sigma_{p}}\right]=-1, \quad d_{\bar{p}}\left(x^{-\sigma_{p}}\right)=1, \quad d_{p}\left(x^{\lambda_{p}}\right)=e_{p} x^{\sigma_{p}+\lambda_{p}}, \quad d_{\bar{p}}\left(x^{\lambda_{p}}\right)=0 .
$$

Thus by replacing $d$ by $d-a_{p} d_{p}-a_{\bar{p}} d_{\bar{p}}$ for some $a_{p}, a_{\bar{p}} \in \mathbb{F}$, we can suppose

$$
c_{-\sigma_{p}, 0}^{\left(\sigma_{p}, 0\right)}=c_{\lambda_{p}, 0}^{\left(\sigma_{p}, \lambda_{p}\right)}=c_{\lambda_{p}, 0}^{(0,0)}=0,
$$

Note again that the replacement does not affect the results we have obtained so far.

Let $q \in I_{1}, q \neq p$. We have

$$
\begin{aligned}
& 0=d\left(\left[x^{-\sigma_{p}}, x^{-\sigma_{q}}\right]\right)=\sum\left(c_{-\sigma_{p}, 0}^{(\beta, j)}\left(\beta_{\bar{q}}-\beta_{q}\right)+c_{-\sigma_{q}, 0}^{\left(\beta+\sigma_{q}-\sigma_{p}, \underline{j}\right)}\left(\beta_{p}-\beta_{\bar{p}}\right)\right) x^{-\sigma_{p}+\beta, \underline{j}}, \\
& 0=d\left(\left[x^{-\sigma_{p}}, x^{\lambda_{q}}\right]\right)=\sum\left(c_{-\sigma_{p}, 0}^{(\beta, j)} \beta_{q} e_{q}+c_{\lambda_{q}, 0}^{\left(\beta+\sigma_{q}-\sigma_{p}, \underline{j}\right)}\left(\beta_{p}-\beta_{\bar{p}}\right)\right) x^{-\sigma_{p}+\sigma_{q}+\beta+\lambda_{q}, \underline{j}} .
\end{aligned}
$$

Now (4.23), (4.34) and (4.35) show that $\beta_{\bar{q}}=\beta_{q}=0$ if $(\beta, \underline{j}) \in M_{-\sigma_{p}}$. Similarly, we can prove $\beta_{r}=j_{r}=0$ for all $r \in J, r \neq p, \bar{p}$ if $(\beta, \underline{j}) \in M_{-\sigma_{p}}$. This and (4.30) show that $(\beta, \underline{j})=\left(\sigma_{p}, 0\right)$ if $(\beta, \underline{j}) \in M_{-\sigma_{p}}$. But $\left(\sigma_{p}, 0\right) \notin M_{-\sigma_{p}}$ by (4.33), i.e., $M_{-\sigma_{p}}=\emptyset$. Thus $d\left(x^{-\sigma_{p}}\right)=0$. Similarly $d\left(x^{\lambda_{p}}\right)=0$. Analogously, we can obtain other results of Claim 3.

Claim 4. We can suppose $d=0$.

Note that $x^{\alpha}$ is a common eigenvector for the elements of the set

$$
A=\left\{x^{-\sigma_{p}}, x^{\varepsilon_{q}}, t_{r} \mid p \in I_{1,4}, q \in I_{5,6}, r \in \bar{I}_{5,6} \cup J_{7}\right\} .
$$

Since $d(A)=0, d\left(x^{\alpha}\right)$ is also a common eigenvector for the elements of $A$. From this and Lemma 3.2 , we obtain

$$
\eta_{\bar{p}} \beta_{p}+\beta_{\bar{p}}=\beta_{q}=0, \underline{j}=0 \text { for } p \in I_{1,4}, q \in I_{5,6} \text { and }(\beta, \underline{j}) \in M_{\alpha} .
$$

For simplicity, we denote $c_{\alpha}^{(\beta)}=c_{\alpha, 0}^{(\beta, 0)}$. We want to prove

$$
d\left(x^{\alpha}\right)=m_{\alpha} x^{\alpha} \text { for } \alpha \in \Gamma \text { and some } m_{\alpha} \in \mathbb{F},
$$


i.e., $M_{\alpha}$ is either empty or a singleton $\{(0,0)\}$. Thus assume that

$$
\beta_{p} \neq 0 \text { for some }(\beta, \underline{j}) \in M_{\alpha}, p \in I_{1,4}, \alpha \in \Gamma \text {. }
$$

For convenience, we again suppose $p \in I_{1}$. Denote $\Gamma_{p}=\left(\mathbb{F} \varepsilon_{p}+\mathbb{F} \varepsilon_{\bar{p}}\right) \cap \Gamma$ as in (3.102), and set $\mathcal{H}_{p}=\operatorname{span}\left\{x^{\alpha} \mid \alpha \in \Gamma_{p}\right\}$. We have

$$
d\left(x^{\alpha}\right) \in \mathcal{H}_{p} \quad \text { for } \quad \alpha \in \Gamma_{p}
$$

by using the fact that $x^{\alpha}$ commutes with elements of $A$ except possibly $x^{-\sigma_{p}}, x^{\lambda_{p}}$. By (4.37) and by

$$
0=d\left(\left[x^{-\sigma_{p}-\lambda_{p}}, x^{\lambda_{p}}\right]\right)=\left[d\left(x^{-\sigma_{p}-\lambda_{p}}\right), x^{\lambda_{p}}\right]=\sum c_{-\sigma_{p}-\lambda_{p}}^{(\beta)} e_{p} x^{\beta},
$$

and by (4.40), we obtain

$$
d\left(x^{-\sigma_{p}-\lambda_{p}}\right)=a_{p} x^{-\lambda_{p}}, \quad d\left(x^{\lambda_{p}-\sigma_{p}}\right)=-a_{p} x^{\lambda_{p}} \quad \text { for some } a_{p} \in \mathbb{F},
$$

where the second equation is obtained from $d\left(\left[x^{-\sigma_{p}-\lambda_{p}}, x^{\lambda_{p}-\sigma_{p}}\right]\right)=0$. Applying $d$ to

$$
\begin{gathered}
{\left[x^{-2 \sigma_{p}}, x^{\lambda_{p}}\right]=-2 e_{p} x^{-\sigma_{p}+\lambda_{p}}, \quad\left[x^{-2 \sigma_{p}}, x^{-\sigma_{p}-\lambda_{p}}\right]=2 e_{p} x^{-2 \sigma_{p}-\lambda_{p}},} \\
{\left[x^{-2 \sigma_{p}-\lambda_{p}}, x^{\lambda_{p}-\sigma_{p}}\right]=-3 e_{p} x^{-2 \sigma_{p}},}
\end{gathered}
$$

we obtain respectively

$$
d\left(x^{-2 \sigma_{p}}\right)=-2 a_{p} x^{-\sigma_{p}}, \quad d\left(x^{-2 \sigma_{p}-\lambda_{p}}\right)=0, \quad a_{p}=0 .
$$

Thus all equations in (4.42) and (4.45) are zero. Applying $d$ to $\left[x^{\lambda_{p}-\sigma_{p}}, x^{k \lambda_{p}}\right]=-k x^{(k+1) \lambda_{p}}$, using induction on $k$, we obtain

$$
d\left(x^{k \lambda_{p}}\right)=0 \quad \text { for } \quad k \geq 1
$$

Applying $d$ to

$$
\begin{gathered}
{\left[x^{\alpha}, x^{-2 \sigma_{p}}\right]=2\left(\alpha_{\bar{p}}-\alpha_{p}\right) x^{\alpha-\sigma_{p}}, \quad\left[x^{\alpha}, x^{-\sigma_{p}-k \lambda_{p}}\right]=\left(\alpha_{\bar{p}}-\alpha_{p}-k \alpha_{\bar{p}} e_{p}\right) x^{\alpha-k \lambda_{p}},} \\
{\left[x^{\alpha-k \lambda_{p}}, x^{k \lambda_{p}}\right]=k \alpha_{p} e_{p} x^{\alpha+\sigma_{p}},}
\end{gathered}
$$

for $k \geq 1$, using (4.37), we obtain

$$
\begin{gathered}
2\left(\alpha_{\bar{p}}-\alpha_{p}\right) c_{\alpha}^{(\beta)}=2\left(\alpha_{\bar{p}}-\alpha_{p}\right) c_{\alpha+\sigma_{p}}^{(\beta)}, \\
\left(\alpha_{\bar{p}}-\alpha_{p}-k\left(\alpha_{p}+\beta_{p}\right) e_{p}\right) c_{\alpha}^{(\beta)}=\left(\alpha_{\bar{p}}-\alpha_{p}-k \alpha_{p} e_{p}\right) c_{\alpha-k \lambda_{p}}^{(\beta)}, \\
k\left(\alpha_{p}+\beta_{p}\right) e_{p} c_{\alpha-k \lambda_{p}}^{(\beta)}=k \alpha_{p} e_{p} c_{\alpha+\sigma_{p}}^{(\beta)} .
\end{gathered}
$$

If $\alpha_{p} \neq \alpha_{\bar{p}}$, then the above three equations gives $\beta_{p}=0$, a contradiction with (4.39). Thus we obtain

$$
\beta_{p} \neq 0,(\beta, 0) \in M_{\alpha} \Rightarrow \alpha_{p}=\alpha_{\bar{p}} .
$$


Replacing $\alpha$ by $\alpha-\sigma_{p}$ in (4.51), it gives

$$
\left(\alpha_{p}-1\right) c_{\alpha}^{(\beta)}=\left(\alpha_{p}-1+\beta_{p}\right) c_{\alpha-\sigma_{p}-\lambda_{p}}^{(\beta)}
$$

Noting that for $\alpha^{\prime}=\alpha-\sigma_{p}-\lambda_{p}$, we have $\alpha_{p}^{\prime} \neq \alpha_{\bar{p}}^{\prime}$. Assume $(\beta, 0) \in M_{\alpha}$. If $(\beta, 0) \in M_{\alpha^{\prime}}$, then (4.52) shows that $\beta_{p}=0$, again a contradiction with (4.39). Thus $(\beta, 0) \notin M_{\alpha^{\prime}}$ and the right-hand side of (4.53) is zero. This and (4.52) show that $\alpha_{\bar{p}}=\alpha_{p}=1$. Note that for $\alpha^{\prime \prime}=\alpha-k \lambda_{p}, k \geq 1$, the relation $\alpha_{\bar{p}}^{\prime \prime}=\alpha_{p}^{\prime \prime}=1$ does not hold, thus the right-hand side of (4.50) is zero. We obtain $\alpha_{p}+\beta_{p}=0$. Hence

$$
\alpha_{\bar{p}}=\alpha_{p}=-\beta_{p}=-\beta_{\bar{p}}=1 \text { if } \beta_{p} \neq 0,(\beta, 0) \in M_{\alpha} .
$$

If $\alpha_{J_{1,4}} \neq \sigma$ (cf. $(2.22)$ ), say $\left(\alpha_{q}, \alpha_{\bar{q}}\right) \neq(1,1)$ for some $q \in I_{1}, q \neq p$. Suppose $\alpha_{q} \neq 1$ (the proof is similar if $\alpha_{\bar{q}} \neq 1$ ), then we can write

$$
x^{\alpha}=\left(\left(\alpha_{q}-1\right) e_{q}\right)^{-1}\left[x^{\alpha-\sigma_{q}-\lambda_{q}+\sigma_{p}}, x^{\lambda_{q}-\sigma_{p}}\right] .
$$

Since for $\alpha^{\prime}=\alpha-\sigma_{q}-\lambda_{q}+\sigma_{p}$ or $\lambda_{q}-\sigma_{p}$, the relation $\alpha_{p}^{\prime}=\alpha_{\bar{p}}^{\prime}=1$ does not holds, we have $\beta_{p}=0$ if $(\beta, 0) \in M_{\alpha^{\prime}}$. Then applying $d$ to (4.55) gives that $\beta_{p}=0$ if $(\beta, 0) \in M_{\alpha}$, which contradicts (4.39) again. Hence

$$
\alpha_{J_{1,4}}=\sigma, \text { and } \beta=-\sigma,
$$

by (4.37) and (4.54). If $\ell_{5}+\ell_{6}+\ell_{7} \neq 0$, we can write

$$
x^{\alpha}= \begin{cases}\left(\alpha_{q}+k\right)^{-1}\left[x^{\alpha+\sigma_{p}+k \varepsilon_{q}}, x^{\left.-\sigma_{p}-k \varepsilon_{q}, \varepsilon_{\bar{q}}\right]}\right. & \text { for } q \in I_{5,6}, k \in \mathbb{Z}, \alpha_{q}+k \neq 0, \\ {\left[x^{\alpha+\sigma_{p}, \varepsilon_{r}}, x^{-\sigma_{p}, \varepsilon_{\bar{r}}}\right]} & \text { for } r \in I_{7} .\end{cases}
$$

Note that for

$$
\left(\alpha^{\prime}, \underline{i}^{\prime}\right)=\left(\alpha+\sigma_{p}+k \varepsilon_{q}, 0\right),\left(-\sigma_{p}-k \varepsilon_{q}, \varepsilon_{\bar{q}}\right),\left(\alpha+\sigma_{p}, \varepsilon_{r}\right) \text { or }\left(-\sigma_{p}, \varepsilon_{\bar{r}}\right),
$$

the relation $\alpha_{p}^{\prime}=\alpha_{\bar{p}}^{\prime}=1$ does not holds, one can prove as above that $\beta_{p}=0$ if $(\beta, 0) \in M_{\alpha^{\prime}, \underline{i}^{\prime}}$. Then applying $d$ to (4.57) gives that $\beta_{p}=0$ if $(\beta, 0) \in M_{\alpha}$, which again contradicts (4.39). Hence $\ell_{5}+\ell_{6}+\ell_{7}=0$. Similarly, one can prove $\ell_{2}+\ell_{3}+\ell_{4}=0$. But then $\iota_{7}=\ell_{1}$, and we can replace $d$ by $d-c_{\alpha}^{(\beta)} d_{0}^{\prime}$ (cf. (4.4) and (4.56)), so that $c_{\alpha}^{(\beta)}$ becomes zero. This proves that the assumption (4.39) does not holds. Thus we have (4.38).

Now we prove

$$
d\left(t^{\underline{i}}\right)=0 \quad \text { if } \quad \underline{i}=\underline{i}_{J_{7}} .
$$

By Claim 1, we can suppose $|\underline{i}|=n \geq 2$. Assume that we have proved (4.59) for $|\underline{i}|<n$. Then $d\left(\left[v, t^{\underline{i}}\right]\right)=0$ for $v \in A$. From this, we obtain $d\left(t^{\underline{i}}\right) \in \mathbb{F} 1_{\mathcal{H}}$. Suppose $i_{p}>0$ for some $p \in I_{7}$. Then $t^{\underline{i}}=\left(i_{\bar{p}}+1\right)^{-1}\left[t_{p}^{2}, t^{\underline{i}-\varepsilon_{p}+\varepsilon_{\bar{p}}}\right]$, and $\left|\underline{i}-\varepsilon_{p}+\varepsilon_{\bar{p}}\right|=n$, thus $d\left(t^{\underline{i}}\right) \in\left[\mathbb{F}, t^{-}-\varepsilon_{p}+\varepsilon_{\bar{p}}\right]+\left[t_{p}^{2}, \mathbb{F}\right]=0$. Similarly, by replacing $d$ by $d-d^{\prime}$ for some $d^{\prime} \in \sum_{q \in I_{2,3} \cup J_{4}} \mathbb{F} \partial_{t_{q}}$ (which does not affect the results we have obtained so far), we can suppose

$$
d\left(x^{\alpha, \underline{i}}\right)=m_{\alpha} x^{\alpha, \underline{i}} \quad \text { for } \quad \alpha \in \Gamma, \underline{i}=\underline{i}_{J_{7}},
$$


and

$$
d\left(t_{p}\right)=d\left(t_{q}^{2}\right)=0 \quad \text { for } \quad p \in I_{2,3} \cup J_{4}, q \in \bar{I}_{5} \cup J_{6} .
$$

Note that $\mathcal{H}$ is generated by elements in (4.60) and (4.61), thus we obtain that (4.60) holds for all $(\alpha, \underline{i}) \in \Gamma \times \mathcal{J}$. From this and (3.1), one can easily deduce that

$$
\mu: \alpha \mapsto m_{\alpha} \text { is a group homomorphism such that } \mu \in \operatorname{Hom}_{\mathbb{Z}}^{+}(\Gamma, \mathbb{F}) \text { if } \iota_{7} \neq \ell_{1} \text {. }
$$

Assume that $\iota_{7}=\ell_{1}$. Then by (3.2) and (4.38), we have

$$
m_{\alpha}+m_{\beta}=m_{\alpha+\beta+\sigma_{p}} \text { if } \alpha_{p} \beta_{\bar{p}} \neq \alpha_{\bar{p}} \beta_{p} \text { and } \alpha, \beta \in \Gamma, p \in I_{1,4} .
$$

By (4.42), (4.45), (4.46), and by induction on $|i|+|j|$, one can prove

$$
m_{i \sigma_{p}+j \lambda_{p}}=0 \quad \text { for } \quad i, j \in \mathbb{Z}, p \in I_{1,4} .
$$

From this we want to prove

$$
m_{\alpha}=m_{\alpha+i \sigma_{p}+j \lambda_{p}} \quad \text { for } \quad \alpha \in \Gamma, i, j \in \mathbb{Z} .
$$

By replacing $\alpha$ by some $\alpha+\sigma_{p}$ if necessary, we can suppose $\left(\alpha_{p}, \alpha_{\bar{p}}\right) \neq(0,0),(1,1)$. By (4.64) and by $\left[x^{\alpha}, x^{-\sigma_{p}+j \lambda_{p}}\right]=\left(\alpha_{p}\left(i+j \lambda_{p}\right)-i \alpha_{\bar{p}}\right) x^{\alpha+(i+1) \sigma_{p}+j \lambda_{p}}$, we obtain $m_{\alpha}=m_{\alpha+i \sigma_{p}+j \lambda_{p}}$ if $\alpha_{p}\left(i-1+j e_{p}\right) \neq(i-1) \alpha_{\bar{p}}$, from this, one can deduce (4.65). Now from (4.63) and (4.65), we obtain (4.62) again. Thus by replacing $d$ by $d-d_{\mu}$, we have $d=0$. This proves Claim 4 and also (4.10).

To prove that (4.11) is a direct sum, suppose

$$
d=a_{0}^{\prime} d_{0}^{\prime}+\sum_{p \in\{0\} \cup J_{1} \cup \bar{I}_{2,3} \cup I_{5}} a_{p} d_{p}+\sum_{q \in I_{2,3} \cup J_{4}} b_{q} \partial_{t_{q}}+d_{\mu}+\sum_{(0,0) \neq(\alpha, \underline{i}) \in \Gamma \times \mathcal{J}} c_{\alpha, \underline{i}} \operatorname{ad}_{x^{\alpha, \underline{i}}},
$$

is the 0 derivation. Applying $d$ to $A \cup\left\{1, t_{p} \mid p \in I_{2,3} \cup J_{4} \cup I_{6}\right\}$ (cf. (4.36)), we obtain that all coefficients are zero except $a_{0}^{\prime}$. Thus $d=a_{0}^{\prime} d_{0}^{\prime}=0$. By (4.4), we obtain either $a_{0}^{\prime}=0$ or $d_{0}^{\prime}=0$. Thus (4.11) is a direct sum.

\section{Second cohomology groups}

In this section, we shall determine the second cohomology groups of the Hamiltonian Lie algebra $\mathcal{H}=\mathcal{H}(\underline{\ell}, \Gamma)$. It is well known that all one-dimensional central extensions of a Lie algebra are determined by the second cohomology group. Central extensions are often used in the structure theory and the representation theory of Kac-Moody algebras [K3]. Using central extension, we can construct many infinite dimensional Lie algebras, such as affine Lie algebras, infinite dimensional Heisenberg algebras, and generalized Virasoro and super-Virasoro algebras, which have a profound mathematical and physical background (cf. [K3, S1, SZ]). Since the cohomology groups are closely related to the structures of Lie algebras, the computation of cohomology groups seems to be important and interesting as well (cf. [J, LW, S1, S2, S3, SZ]).

Recall that a 2-cocycle on $\mathcal{H}$ is an $\mathbb{F}$-bilinear function $\psi: \mathcal{H} \times \mathcal{H} \rightarrow \mathbb{F}$ satisfying the following conditions:

$$
\begin{array}{ll}
\psi\left(v_{1}, v_{2}\right)=-\psi\left(v_{2}, v_{1}\right) & \text { (skew-symmetry), } \\
\psi\left(\left[v_{1}, v_{2}\right], v_{3}\right)+\psi\left(\left[v_{2}, v_{3}\right], v_{1}\right)+\psi\left(\left[v_{3}, v_{1}\right], v_{2}\right)=0 & \text { (Jacobian identity), }
\end{array}
$$


for $v_{1}, v_{2}, v_{3} \in \mathcal{H}$. Denote by $C^{2}(\mathcal{H}, \mathbb{F})$ the vector space of 2-cocycles on $\mathcal{H}$. For any $\mathbb{F}$-linear function $f: \mathcal{H} \rightarrow \mathbb{F}$, one can define a 2-cocycle $\psi_{f}$ as follows

$$
\psi_{f}\left(v_{1}, v_{2}\right)=f\left(\left[v_{1}, v_{2}\right]\right) \text { for } v_{1}, v_{2} \in \mathcal{H} \text {. }
$$

Such a 2-cocycle is called a 2-coboundary or a trivial 2-cocycle on $\mathcal{H}$. Denote by $B^{2}(\mathcal{H}, \mathbb{F})$ the vector space of 2-coboundaries on $\mathcal{H}$. A 2-cocycle $\phi$ is said to be equivalent to a 2-cocycle $\psi$ if $\phi-\psi$ is trivial. For a 2-cocycle $\psi$, we denote by $[\psi]$ the equivalent class of $\psi$. The quotient space

$$
H^{2}(\mathcal{H}, \mathbb{F})=C^{2}(\mathcal{H}, \mathbb{F}) / B^{2}(\mathcal{H}, \mathbb{F})=\{\text { the equivalent classes of 2-cocycles }\},
$$

is called the second cohomology group of $\mathcal{H}$.

Lemma 5.1. If $\iota_{7} \neq \ell_{1}$, then $H^{2}(\mathcal{H}, \mathbb{F})=0$.

Proof. Let $\psi$ be a 2-cocycle. Say $\ell_{4} \neq 0$ (the proof is exactly similar if $\ell_{i} \neq 0$ for $i \neq 1,4$ ). We fix $p \in I_{4}$. Define a linear function $f$ by induction on $i_{\bar{p}}$ as follows:

$$
f\left(x^{\alpha, \underline{i}}\right)= \begin{cases}\alpha_{\bar{p}}^{-1}\left(\psi\left(t_{p}, x^{\alpha, \underline{i}}\right)-i_{\bar{p}} f\left(x^{\alpha, \underline{i}-\varepsilon_{\bar{p}}}\right)\right) & \text { if } \alpha_{\bar{p}} \neq 0 \\ \left(i_{\bar{p}}+1\right)^{-1} \psi\left(t_{p}, x^{\alpha, \underline{i}+\varepsilon_{\bar{p}}}\right) & \text { if } \alpha_{\bar{p}}=0\end{cases}
$$

for $(\alpha, \underline{i}) \in \Gamma \times \mathcal{J}$. Set $\phi=\psi-\psi_{f}$. Then (5.5) shows that

$$
\phi\left(t_{p}, x^{\alpha, \underline{i}}\right)=0 \quad \text { for } \quad(\alpha, \underline{i}) \in \Gamma \times \mathcal{J} .
$$

Using Jacobian identity (5.2), we obtain

$$
0=\phi\left(t_{p},\left[x^{\alpha, \underline{i}}, x^{\beta, \underline{j}}\right]\right)=\left(\alpha_{\bar{p}}+\beta_{\bar{p}}\right) \phi\left(x^{\alpha, \underline{i}}, x^{\beta, \underline{j}}\right)+i_{\bar{p}} \phi\left(x^{\alpha, \underline{i}-\varepsilon_{\bar{p}}}, x^{\beta, \underline{j}}\right)+j_{\bar{p}} \phi\left(x^{\alpha, \underline{i}}, x^{\beta, \underline{j}-\varepsilon_{\bar{p}}}\right),
$$

for $(\alpha, \underline{i}),(\beta, \underline{j}) \in \Gamma \times \mathcal{J}$. If $\alpha_{\bar{p}}+\beta_{\bar{p}} \neq 0$, by induction on $i_{\bar{p}}+j_{\bar{p}}$, we obtain $\phi\left(x^{\alpha, \underline{i}}, x^{\beta, \underline{j}}\right)=0$. On the other hand, if $\alpha_{\bar{p}}+\beta_{\bar{p}}=0$, then (5.7) gives

$$
\phi\left(x^{\alpha, \underline{i}}, x^{\beta, \underline{j}}\right)=-j_{\bar{p}}\left(i_{\bar{p}}+1\right)^{-1} \phi\left(x^{\alpha, \underline{i}+\varepsilon_{\bar{p}}}, x^{\beta, \underline{j}-\varepsilon_{\bar{p}}}\right),
$$

and by induction on $j_{\bar{p}}$, we again have $\phi\left(x^{\alpha, \underline{i}}, x^{\beta, \underline{j}}\right)=0$. Thus $\phi=0$.

Assume that $\iota_{7}=\ell_{1}$. Denote $\sigma=\sum_{p \in I_{1}} \sigma_{p}$, and we use notation $\operatorname{Hom}_{\mathbb{Z}}^{*}(\Gamma, \mathbb{F})$ as in (4.9) (cf. (4.6)). We construct 2-cocycles $\phi_{p}, \phi_{p}^{\prime}, \phi_{\mu}$ for $p \in I_{1}, \mu \in \operatorname{Hom}_{\mathbb{Z}}^{*}(\Gamma, \mathbb{F})$ as follows:

$$
\begin{aligned}
& \phi_{p}\left(x^{\alpha}, x^{\beta}\right)=\alpha_{p} \delta_{\alpha+\beta, \sigma-\sigma_{p}}, \\
& \phi_{p}^{\prime}\left(x^{\alpha}, x^{\beta}\right)=\alpha_{\bar{p}} \delta_{\alpha+\beta, \sigma-\sigma_{p}}, \\
& \phi_{\mu}\left(x^{\alpha}, x^{\beta}\right)=\mu(\alpha) \delta_{\alpha+\beta, \sigma},
\end{aligned}
$$

for $\alpha, \beta \in \Gamma$. It is straightforward to verify that they are 2-cocycles (cf. $[\mathrm{J}]$ ). From the proof of Theorem 5.2 below, one can see why we construct such 2-cocycles.

Theorem 5.2. (1) $H^{2}(\mathcal{H}, \mathbb{F})=0$ if $\iota_{7} \neq \ell_{1}$; (2) if $\iota_{7}=\ell_{1}$, then $H^{2}(\mathcal{H}, \mathbb{F})$ is the vector space spanned by $B=\left\{\left[\phi_{p}\right],\left[\phi_{p}^{\prime}\right],\left[\phi_{\mu}\right] \mid p \in I_{1}, \mu \in \operatorname{Hom}_{\mathbb{Z}}^{*}(\Gamma, \mathbb{F})\right\}$. Furthermore, for $a_{p}, b_{p} \in \mathbb{F}, \mu \in$ $\operatorname{Hom}_{\mathbb{Z}}^{*}(\Gamma, \mathbb{F})$, we have

$$
\sum_{p \in I_{1}}\left(a_{p}\left[\phi_{p}\right]+b_{p}\left[\phi_{p}^{\prime}\right]\right)+\left[\phi_{\mu}\right]=0 \Leftrightarrow a_{p}=b_{p}=\mu=0 .
$$


Proof. (1) follows from Lemma 5.1, while (2) follows from [J]. We give a simple proof of (2) as follows.

First we prove (5.12). Thus suppose

$$
\psi=\sum_{p \in I_{1}}\left(a_{p} \phi_{p}+b_{p} \phi_{p}^{\prime}\right)+\phi_{\mu}+\psi_{f}
$$

is the zero 2-cocycle for some $a_{p}, b_{p} \in \mathbb{F}$ and some linear function $f$. Then for $p \in I_{1}, \alpha \in \Gamma$, by applying $\psi$ to $\left(x^{-\sigma_{p}}, x^{\sigma}\right),\left(x^{\lambda_{p}}, x^{\sigma-\lambda_{p}-\sigma_{p}}\right),\left(x^{\alpha}, x^{\sigma-\alpha}\right)$, we have

$$
\begin{aligned}
& 0=\psi\left(x^{-\sigma_{p}}, x^{\sigma}\right)=-a_{p}-b_{p}, \\
& 0=\psi\left(x^{\lambda_{p}}, x^{\sigma-\lambda_{p}-\sigma_{p}}\right)=e_{p} b_{p}, \\
& 0=\psi\left(x^{\alpha}, x^{\sigma-\alpha}\right)=\mu(\alpha)+\sum_{p \in I_{1}}\left(\alpha_{p}-\alpha_{\bar{p}}\right) f\left(x^{\sigma_{p}+\sigma}\right),
\end{aligned}
$$

(cf. the definition of $\lambda_{p}$ in (3.97)). We obtain that $a_{p}=b_{p}=0$ for $p \in I_{1}$ and by (4.9),

$$
\mu=\sum_{p \in I_{1}} c_{p} \mu_{p} \in \operatorname{Hom}_{\mathbb{Z}}^{*}(\Gamma, \mathbb{F}) \cap \operatorname{span}\left\{\mu_{p} \mid p \in I_{1}\right\}=\{0\},
$$

where $c_{p}=-f\left(x^{\sigma_{p}+\sigma}\right) \in \mathbb{F}$. This proves (5.12).

Now suppose $\psi$ is a 2-cocycle. We define a linear function $f$ as follows: set $f\left(x^{\sigma}\right)=0$, and for $\alpha \in \Gamma \backslash\{\sigma\}$, we define

$$
p_{\alpha}=\min \left\{p \in I_{1} \mid\left(\alpha_{p}, \alpha_{\bar{p}}\right) \neq(1,1)\right\}
$$

and set

$$
f\left(x^{\alpha}\right)= \begin{cases}\left(\alpha_{p}-\alpha_{\bar{p}}\right)^{-1} \psi\left(x^{-\sigma_{p}}, x^{\alpha}\right) & \text { if } \alpha_{\bar{p}} \neq \alpha_{p} \\ e_{p}^{-1}\left(1-\alpha_{p}\right)^{-1} \psi\left(x^{\lambda_{p}}, x^{\alpha-\sigma_{p}-\lambda_{p}}\right) & \text { if } \alpha_{\bar{p}}=\alpha_{p} \neq 1\end{cases}
$$

for $p=p_{\alpha}$. Set

$$
\phi=\psi-\sum_{p \in I_{1}}\left(a_{p} \phi_{p}+b_{p} \phi_{p}^{\prime}\right)-\psi_{f}
$$

where

$$
a_{p}=-\psi\left(x^{-\sigma_{p}}, x^{\sigma}\right)-b_{p}, \quad b_{p}=e_{p}^{-1} \psi\left(x^{\lambda_{p}}, x^{\sigma-\lambda_{p}-\sigma_{p}}\right),
$$

(cf. (5.14) and (5.15)). Then one can prove

$$
\phi\left(x^{-\sigma_{p}}, x^{\alpha}\right)=0 \text { for } p \in I_{1}, \alpha \in \Gamma \text {. }
$$

In fact, if $\alpha=\sigma$, it follows from (5.9), (5.10), (5.20) and (5.21). Assume $\alpha \neq \sigma$. Let $p=p_{\alpha}$ and write

$$
x^{\alpha}= \begin{cases}\left(\alpha_{p}-\alpha_{\bar{p}}\right)^{-1}\left[x^{-\sigma_{p}}, x^{\alpha}\right] & \text { if } \alpha_{\bar{p}} \neq \alpha_{p}, \\ e_{p}^{-1}\left(1-\alpha_{p}\right)^{-1}\left[x^{\lambda_{p}}, x^{\alpha-\sigma_{p}-\lambda_{p}}\right] & \text { if } \alpha_{\bar{p}}=\alpha_{p} \neq 1,\end{cases}
$$

(cf. (5.19)), we can obtain (5.22) by the Jacobian identity (5.2). From (5.22), by considering $\phi\left(x^{-\sigma_{p}},\left[x^{\alpha}, x^{\beta}\right]\right)$ and by $(5.2)$, we obtain

$$
\phi\left(x^{\alpha}, x^{\beta}\right)=0 \quad \text { if } \quad \alpha_{p}+\beta_{p} \neq \alpha_{\bar{p}}+\beta_{\bar{p}} \quad \text { for some } p \in I_{1} .
$$


Now we want to prove

$$
\phi\left(x^{\lambda_{q}}, x^{\alpha}\right)=0 \quad \text { for } \quad \alpha \in \Gamma, q \in I_{1} .
$$

By (5.24), we can suppose $\alpha_{p}=\alpha_{\bar{p}}$ if $p \neq q$, and $\alpha_{q}=\alpha_{\bar{q}}+e_{q}$. If $\alpha=\sigma-\lambda_{q}-\sigma_{q}$, (5.25) follows from (5.9), (5.10), (5.20) and (5.21). So suppose $\alpha \neq \sigma-\lambda_{q}-\sigma_{q}$. Let $\alpha^{\prime}=\alpha+\lambda_{q}+\sigma_{q} \neq \sigma$. If $p=p_{\alpha^{\prime}} \neq q$, then $\alpha_{p}^{\prime}=\alpha_{\bar{p}}^{\prime}=\alpha_{p}=\alpha_{\bar{p}} \neq 1$, and by writing $x^{\alpha}=e_{p}^{-1}\left(1-\alpha_{p}\right)^{-1}\left[x^{\lambda_{p}}, x^{\alpha-\sigma_{p}-\lambda_{p}}\right]$, we obtain

$$
\phi\left(x^{\lambda_{q}}, x^{\alpha}\right)=-e_{p}^{-1}\left(1-\alpha_{p}\right)^{-1} e_{q} \alpha_{q} \phi\left(x^{\lambda_{p}}, x^{\alpha^{\prime}-\sigma_{p}-\lambda_{p}}\right)=0,
$$

by (5.19). On the other hand, if $p=q$, we again have $\phi\left(x^{\lambda_{q}}, x^{\alpha}\right)=\phi\left(x^{\lambda_{q}}, x^{\alpha^{\prime}-\sigma_{p}-\lambda_{p}}\right)=0$ by (5.19).

Now by $(5.25)$ and by writing $\left(\alpha_{p}-1\right) x^{\alpha}=-e_{p}^{-1}\left[x^{\lambda_{p}}, x^{\alpha-\lambda_{p}-\sigma_{p}}\right]$, we obtain

$$
\begin{gathered}
\left(\alpha_{p}-1\right) \phi\left(x^{\alpha}, x^{\beta}\right)=-\beta_{p} \phi\left(x^{\alpha-\lambda_{p}-\sigma_{p}}, x^{\beta+\lambda_{p}+\sigma_{p}}\right) \\
\left(\alpha_{p}-2\right) \phi\left(x^{\alpha-\lambda_{p}-\sigma_{p}}, x^{\beta+\lambda_{p}+\sigma_{p}}\right)=-\left(\beta_{p}+1\right) \phi\left(x^{\alpha-2 \lambda_{p}-2 \sigma_{p}}, x^{\beta+2 \lambda_{p}+2 \sigma_{p}}\right),
\end{gathered}
$$

for $p \in I_{1}$, where (5.28) is obtained from (5.27). Using (5.28) in (5.27) and by writing

$$
\left(3\left(\alpha_{\bar{p}}-\alpha_{p}\right)-2 \alpha_{p} e_{p}\right) x^{\alpha-2 \lambda_{p}-2 \sigma_{p}}=\left[x^{\alpha}, x^{-2 \lambda_{p}-3 \sigma_{p}}\right],
$$

we obtain

$$
\begin{aligned}
& \left(3\left(\alpha_{\bar{p}}-\alpha_{p}\right)-2 \alpha_{p} e_{p}\right)\left(\alpha_{p}-1\right)\left(\alpha_{p}-2\right) \phi\left(x^{\alpha}, x^{\beta}\right) \\
& \quad=\beta_{p}\left(\beta_{p}+1\right)\left(\phi\left(\left[x^{\alpha}, x^{\beta+2 \lambda_{p}+2 \sigma_{p}}\right], x^{-2 \lambda_{p}-3 \sigma_{p}}\right)+\phi\left(x^{\alpha},\left[x^{-2 \lambda_{p}-3 \sigma_{p}}, x^{\beta+2 \lambda_{p}+2 \sigma_{p}}\right]\right)\right) .
\end{aligned}
$$

We prove that

$$
\phi\left(x^{\alpha}, x^{-2 \lambda_{p}-3 \sigma_{p}}\right)=0 \quad \text { for } \quad \alpha \in \Gamma, p \in I_{1} .
$$

By (5.24), we can suppose $\alpha_{\bar{p}}=\alpha_{p}+2 e_{p}$. If $\alpha_{p} \neq 1,2$, by setting $\beta=-2 \lambda_{p}-3 \sigma_{p}$ in (5.27) and $(5,28)$, then the right-hand side of (5.28) is zero by (5.22), and thus (5.31) holds. Suppose $\alpha_{p}=1,2$. Then $\alpha_{\bar{p}}=1+2 e_{p}$ or $2+2 e_{p}$, thus we can write $\alpha$ in the following form

$$
\alpha=\alpha^{\prime}+\sigma_{p}+2 \lambda_{p} \text { or } \alpha^{\prime}+2 \sigma_{p}+2 \lambda_{p} \text { for some } \alpha^{\prime} \in \Gamma \text { such that }\left(\alpha_{p}^{\prime}, \alpha_{\bar{p}}^{\prime}\right)=(0,0) .
$$

We denote

$$
\begin{array}{ll}
c_{i}=\phi\left(x^{\alpha^{\prime}+i\left(\sigma_{p}+\lambda_{p}\right)-\sigma_{p}}, x^{-i\left(\sigma_{p}+\lambda_{p}\right)-\sigma_{p}}\right), & c_{i}^{\prime}=\phi\left(x^{\alpha^{\prime}+i\left(\sigma_{p}+\lambda_{p}\right)}, x^{-i\left(\sigma_{p}+\lambda_{p}\right)-\sigma_{p}}\right), \\
d_{i}=\phi\left(x^{\alpha^{\prime}+i\left(\sigma_{p}+\lambda_{p}\right)-2 \sigma_{p}}, x^{-i\left(\sigma_{p}+\lambda_{p}\right)}\right), & d_{i}^{\prime}=\phi\left(x^{\alpha^{\prime}+i\left(\sigma_{p}+\lambda_{p}\right)-\sigma_{p}}, x^{-i\left(\sigma_{p}+\lambda_{p}\right)}\right),
\end{array}
$$

for $i \in \mathbb{Z}$. By writing

$$
(i-2 j) e_{p} x^{-i\left(\sigma_{p}+\lambda_{p}\right)-\sigma_{p}}=\left[x^{-j\left(\sigma_{p}+\lambda_{p}\right)-\sigma_{p}}, x^{-(i-j)\left(\sigma_{p}+\lambda_{p}\right)-\sigma_{p}}\right] \quad \text { for } \quad j \in \mathbb{Z},
$$

we obtain

$$
(i-2 j) c_{i}=(i+j) c_{i-j}-(2 i-j) c_{j}, \quad(i-2 j) c_{i}^{\prime}=i\left(c_{i-j}^{\prime}-c_{j}^{\prime}\right) \quad \text { for } \quad i, j \in \mathbb{Z}
$$

By writing

$$
\begin{aligned}
& 2(j-i) e_{p} x^{\alpha^{\prime}+i\left(\sigma_{p}+\lambda_{p}\right)-\sigma_{p}}=\left[x^{\alpha^{\prime}+j\left(\sigma_{p}+\lambda_{p}\right)-2 \sigma_{p}}, x^{(i-j)\left(\sigma_{p}+\lambda_{p}\right)}\right], \\
& (j-i) e_{p} x^{\alpha^{\prime}+i\left(\sigma_{p}+\lambda_{p}\right)}=\left[x^{\alpha^{\prime}+j\left(\sigma_{p}+\lambda_{p}\right)-\sigma_{p}}, x^{(i-j)\left(\sigma_{p}+\lambda_{p}\right)}\right],
\end{aligned}
$$


we obtain

$$
2(j-i) c_{i}=(2 i+j) d_{j-i}-(i-j) d_{j}, \quad(j-i) c_{i}^{\prime}=(i+j) d_{j-i}^{\prime}+(i-j) d_{j}^{\prime} \quad \text { for } \quad i, j \in \mathbb{Z} .
$$

Note that the system (5.36) has up to multiplicative scalars unique solutions for $c_{i}, c_{i}^{\prime}$, and we find that

$$
c_{i}=\left(i^{3}-i\right) c, \quad c_{i}^{\prime}=i^{2} c^{\prime} \quad \text { for } i \in \mathbb{Z} \text { and some } c, c^{\prime} \in \mathbb{F},
$$

are the only solutions. If we substitute $j$ by 1 and by $i+1$ in (5.39), we then obtain $c_{i}=c_{i}^{\prime}=$ $d_{i}=d_{i}^{\prime}=0$ for all $i \in \mathbb{Z}$. This in particular proves (5.31) by (5.32)-(5.34).

Now using (5.31) in (5.30), noting that $\beta_{p}-\beta_{\bar{p}}=\alpha_{\bar{p}}-\alpha_{p}$ by (5.24), we deduce that

$$
\left(\beta_{p}\left(\beta_{p}+1\right)\left(3\left(\alpha_{\bar{p}}-\alpha_{p}\right)+2 e_{p}\left(\beta_{p}-1\right)\right)-\left(\alpha_{p}-1\right)\left(\alpha_{p}-2\right)\left(3\left(\alpha_{\bar{p}}-\alpha_{p}\right)-2 \alpha_{p} e_{p}\right)\right) \phi\left(x^{\alpha}, x^{\beta}\right)=0 .
$$

As in the proof of $(5.31)$, we can prove $\phi\left(x^{\alpha}, x^{2 \lambda_{p}}\right)=0$. Thus we can replace $\lambda_{p}$ by $2 \lambda_{p}$ in the above discussion, i.e., if we replace $e_{p}$ by $2 e_{p},(5.41)$ still holds. This forces

$$
\phi\left(x^{\alpha}, x^{\beta}\right)=0 \text { or } \beta_{p}-1=-\alpha_{p} \text { for all } p \in I_{1} \text {, }
$$

i.e., if $\alpha+\beta \neq \sigma$, then $\phi\left(x^{\alpha}, x^{\beta}\right)=0$. Thus we can suppose

$$
\phi\left(x^{\alpha}, x^{\beta}\right)=m_{\alpha} \delta_{\alpha+\beta, \sigma} \text { for } \alpha, \beta \in \Gamma \text { and some } m_{\alpha} \in \mathbb{F} \text {. }
$$

As in the proof of (5.31), we can prove $m_{i \sigma_{p}+j \lambda_{p}}=0$ for $i, j \in \mathbb{Z}, p \in I_{1}$. Then for any $\alpha, \beta \in \Gamma, p \in I_{1}$, let $v_{1}=x^{\alpha}, v_{2}=x^{\beta}, v_{3}=x^{\sigma-\alpha-\beta-\sigma_{p}}$ in (5.2), one can easily deduce that $\mu: \alpha \mapsto m_{\alpha}$ is a group homomorphism $\mu: \Gamma \rightarrow \mathbb{F}$ such that $\mu\left(\sigma_{p}\right)=0$. Thus $\mu \in \operatorname{Hom}_{\mathbb{Z}}^{+}(\Gamma, \mathbb{F})$ and $\phi=\psi_{\mu}$. Furthermore, we can write $\mu=\nu+\lambda$ for $\nu \in \operatorname{Hom}_{\mathbb{Z}}^{*}(\Gamma, \mathbb{F}), \lambda \in \operatorname{span}\left\{\mu_{p} \mid p \in I_{1}\right\}$ by (4.9). Then $\phi=\psi_{\nu}+\psi_{\lambda}$. But from (5.16) and (5.17), one can see that $\psi_{\lambda}$ corresponds to a trivial 2-cocycle, thus we can suppose $\phi=\phi_{\nu}$. This proves Theorem 5.1.

\section{References}

[DZ] D. Dokovic and K. Zhao, Derivations, isomorphisms and second cohomology of generalized Block algebras, Alg. Colloq. 3 (1996), 245-272.

[F] R. Farnsteiner, Derivations and central extensions of finitely generated graded Lie algebras, J. Alg. 118 (1988), 33-45.

[J] Y. Jia, Second cohomology of generalized Cartan type $H$ Lie algebras in characteristic 0, J. Alg. 204 (1998), 312-323.

[K1] V. G. Kac, A description of filtered Lie algebras whose associated graded Lie algebras are of Cartan types, Math. of USSR-Izvestijia 8 (1974), 801-835.

[K2] V. G. Kac, Classification of infinite-dimensional simple linearly compact Lie superalgebras, Adv. Math. 139 (1998), 1-55.

[K3] V. G. Kac, Infinite Dimensional Lie Algebras, 3rd ed., Cambridge Univ. Press, 1990. 
[LW] W. Li, R. L. Wilson, Central extensions of some Lie algebras, Proc. Amer. Math. Soc. 126 (1998), 2569-2577.

[O] J. M. Osborn, New simple infinite-dimensional Lie algebras of characteristic 0, J. Alg. 185 (1996), 820-835.

[OZ] J. M. Osborn and K. Zhao, Generalized Poisson brackets and Lie algebras for type $H$ in characteristic 0, Math. Z. 230 (1999), 107-143.

[S1] Y. Su, 2-Cocycles on the Lie algebras of generalized differential operators, Comm. Alg. 30 (2002), 763-782.

[S2] Y. Su, On the low dimensional cohomology of Kac-Moody algebras with coefficients in the complex field, Shuxue Jinzhan (Chinese Adv. in Math.) 1989, 18, 346-351.

[S3] Y. Su, 2-Cocycles on the Lie algebras of all differential operators of several indeterminates. (Chinese) Northeastern Math. J. 6 (1990), 365-368.

[SX] Y. Su and X. Xu, Central simple Poisson algebras, to appear.

[SXZ] Y. Su, X. Xu and H. Zhang, Derivation-simple algebras and the structures of Lie algebras of Witt type, J. Alg. 233 (2000), 642-662.

[SZ] Y. Su, K. Zhao, Second cohomology group of generalized Witt type Lie algebras and certain representations, Comm. Alg. 30 (2002), 3285-3309.

[X1] X. Xu, Generalizations of Block algebras, Manuscripta Math. 100 (1999), 489-518.

[X2] X. Xu, New generalized simple Lie algebras of Cartan type over a field with characteristic 0, J. Alg. 224 (2000), 23-58.

[Z] H. Zhang, The representations of the coordinate ring of the quantum symplectic space, J. Pure Appl. Algebra 150 (2000), 95-106.

[Zh] K. Zhao, A class of infinite dimensional simple Lie algebras, J. London Math. Soc. (2), 62 (2000), 71-84. 